\title{
Intraprocedural guidance in percutaneous mitral valve repair
}

\author{
Francesco Ancona1, Stefano Stella1, Cristina Capogrosso, Francesco Melillo', Giacomo Ingallina', \\ Antonio Boccellino', Antonio Napolano', Eustachio Agricola ${ }^{1,2}$ \\ ${ }^{1}$ Cardiovascular Imaging Unit, Cardio-Thoracic-Vascular Department, IRCCS San Raffaele Scientific Institute, Milan 20132, Italy.
} ${ }^{2}$ School of Medicine, Vita-Salute San Raffaele University, Milan 20132, Italy.

Correspondence to: Dr. Francesco Ancona, Cardiovascular Imaging Unit, Cardio-Thoracic-Vascular Department, IRCCS San Raffaele Scientific Institute, Via OIgettina 60, Milan 20132, Italy. E-mail: ancona.francesco@hsr.it

How to cite this article: Ancona F, Stella S, Capogrosso C, Melillo F, Ingallina G, Boccellino A, Napolano A, Agricola E. Intraprocedural guidance in percutaneous mitral valve repair. Mini-invasive Surg 2020;4:79.

http://dx.doi.org/10.20517/2574-1225.2020.80

Received: 6 Aug 2020 First Decision: 21 Sep 2020 Revised: 23 Sep 2020 Accepted: 10 Oct 2020 Published: 6 Nov 2020

Academic Editor: Azeem Latib Copy Editor: Cai-Hong Wang Production Editor: Jing Yu

\begin{abstract}
Percutaneous mitral valve intervention is emerging as a valid alternative for patients affected by mitral regurgitation. By addressing the pathophysiology, therapeutic options mainly target the leaflets, annulus or left ventricle. The present review will cover the intraprocedural guidance of the most used approaches, such as edge to edge repair, adjustable transapical beating-heart chordal implantation and percutaneous direct or indirect annuloplasty. Intraprocedural monitoring relies on integration of fluoroscopy and echocardiography, and is based on the continuous communication between the interventional imager and the interventional cardiologist.
\end{abstract}

Keywords: Mitral regurgitation, transcatheter mitral valve interventions, percutaneous edge to edge mitral valve repair, percutaneous direct mitral valve annuloplasty, interventional echocardiography

\section{INTRODUCTION}

Percutaneous mitral valve intervention is emerging as an alternative for high-risk patients with mitral regurgitation (MR) who are not suitable for conventional open-heart surgery. The current therapeutic options available are classified according to the main physio-pathological targets namely the leaflets, annulus and chordae tendinae.

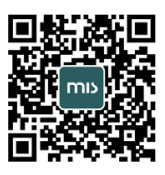


The present review will cover the intraprocedural guidance of the most utilised approaches for mitral valve (MV) repair in the setting of MR, such as (1) leaflet repair: edge to edge repair; (2) chordal repair: adjustable transapical beating-heart chordal implantation; and (3) annular repair: percutaneous direct or indirect annuloplasty.

\section{Baseline intraprocedural evaluation of MR grading}

MR severity varies in a spectrum, especially in functional MR which is load dependent. Hence, it is imperative to obtain a baseline grading prior to all transcatheter procedures ${ }^{[1]}$, in order to extrapolate the procedural results under similar hemodynamic conditions and controlled heart rate. In addition, as the ultrasound machine settings (particularly color scale and gain) could influence evaluation, these should be standardized for pre- and post-device evaluation. A multi-modal approach seems the most suitable and appropriate to quantify MR in this setting ${ }^{[2]}$.

\section{LEAFLET REPAIR: PERCUTANEOUS EDGE-EDGE}

The most widely experienced percutaneous approach to MV repair mimics surgical edge to edge repair ${ }^{[3]}$. Nowadays two devices are currently available: Mitraclip ${ }^{\mathrm{TM}}$ system (Abbott Vascular Inc., Menlo, CA, USA) and PASCAL ${ }^{\mathrm{TM}}$ device (Edwards Life- sciences, Irvine, California).

The crucial procedural steps are the following: (1) safe trans-septal puncture at an optimal site and placement of the guide catheter; (2) steering of the device delivery system (DS) within the LA toward MV plane; (3) perpendicular alignment of DS to the MV coaptation line; (4) creation of the tissue bridge between anterior and posterior leaflet at the target site via grasping and adequate leaflet insertion; (5) evaluation of MR reduction without significant mitral stenosis; and (6) assessment of residual interatrial septal shunt ${ }^{[4-6]}$.

\section{Mitraclip $^{\mathrm{TM}}$ system}

The Mitraclip ${ }^{\mathrm{TM}}$ system consists of a polyester fabric covered cobalt-chromium implant with two arms which can be opened and closed with a steerable-guiding mechanism. Currently there are 2 available devices: NTR and XTR.

\section{Intraprocedural monitoring}

Under general anesthesia, a trans-septal approach is utilized and the DS is aligned perpendicularly to the MV plane and to the coaptation line of MV leaflets. The device is deployed after successfully grasping of leaflets, at the level of the regurgitant target lesion. In degenerative MR, the clip aims to anchor the flail and/or prolapsing segments, whereas, in cases of functional MR, to improve coaptation of the leaflets. If needed, additional clip(s) may be placed.

Echocardiographic (2D/3D TEE) and fluoroscopic imaging are used during the procedure. Procedural steps and relative imaging modalities are summarized in Table $1^{[7]}$.

\section{Transseptal puncture}

The initial and fundamental step is the trans-septal puncture (TSP) [Figure 1]. The determination of the optimal TSP site is of utmost importance for MitraClip ${ }^{\mathrm{TM}}$ procedure, as a suboptimal puncture site often leads to additional steering maneuvers to correct the position of the DS within the left atrium (LA), increasing complexity and duration of the procedure. Moreover, optimal puncture height also depends on MV pathology, particularly in cases of degenerative and secondary MR. In patients with prolapse/flail, the puncture site should be higher ( $\sim 4.5-5 \mathrm{~cm}$ above the mitral annulus), thus providing enough space to adequately maneuver the DS within the LA. In cases of secondary MR with prominent apical tethering of the leaflets, since the coaptation point is usually shifted below the annular plane, a lower puncture site 
Table 1. Mitraclip ${ }^{\mathrm{TM}}$ : Imaging modality for each procedural step

\begin{tabular}{|c|c|c|c|c|}
\hline \multirow{2}{*}{\multicolumn{2}{|c|}{ Procedural step }} & \multicolumn{2}{|c|}{ Imaging modality } & \multirow{2}{*}{ TIPS and TRICKS } \\
\hline & & \multirow[b]{2}{*}{\begin{tabular}{l}
\multicolumn{1}{c}{ Echocardiography } \\
Biplane views: bicaval and SAX \\
views \\
3D lateral perspective of IAS \\
ME 4-chamber view with \\
retroflexion (height)
\end{tabular}} & \multirow{2}{*}{$\begin{array}{l}\text { Fluoroscopy } \\
\text { AP projection } \\
\text { LAO projection }\end{array}$} & \\
\hline 1. & $\begin{array}{l}\text { Tailored Trans-septal } \\
\text { puncture }\end{array}$ & & & $\begin{array}{l}\text { => sharp tenting should be seen } \\
\text { => superior and posterior location in the fossa } \\
\text { with a height of } 4-4.5 \mathrm{~cm} \text { to the annulus (see text } \\
\text { for details) } \\
=>\text { avoid PFO }\end{array}$ \\
\hline 2. & $\begin{array}{l}\text { Steerable guiding } \\
\text { catheter into LA }\end{array}$ & $\begin{array}{l}\text { 2D SAX } \\
\text { 2D LAX } \\
\text { 4-chamber view } \\
\text { 3D overhead of LA } \\
\text { 3D lateral view }\end{array}$ & AP projection & $\begin{array}{l}=>\text { dilator is removed when the SGC is at least } 2 \\
\mathrm{~cm} \text { across the IAS }\end{array}$ \\
\hline 3. & $\begin{array}{l}\text { Clip delivery system } \\
\text { into LA }\end{array}$ & $\begin{array}{l}\text { 3D overhead of } L A \\
2 D \text { ME views }\end{array}$ & AP projection & \\
\hline 4. & $\begin{array}{l}\text { Steering and } \\
\text { Positioning }\end{array}$ & $\begin{array}{l}\text { Biplane views: commissural and } \\
\text { LAX views with and without } \\
\text { color Doppler } \\
\text { 3D overhead of LA }\end{array}$ & RAO CRA & \\
\hline 5. & Axial alignment & $\begin{array}{l}\text { Biplane views: commissural and } \\
\text { LAX views }\end{array}$ & RAO CRA & $\begin{array}{l}=>\text { check perpendicularity (3D) and the path of } \\
\text { clip (biplane) towards the target lesion }\end{array}$ \\
\hline 6. & $\begin{array}{l}\text { Alignment of the Clip } \\
\text { arms }\end{array}$ & $\begin{array}{l}\text { 3D en face view } \\
\text { Biplane views: commissural and } \\
\text { LAX } \\
\text { views } \\
\text { MV SAX transgastric view }\end{array}$ & RAO CRA & $\begin{array}{l}=>\text { clip should be clearly visualized in the LAX } \\
\text { view }\end{array}$ \\
\hline 7. & $\begin{array}{l}\text { Advancement into } \\
\text { LV }\end{array}$ & $\begin{array}{l}\text { Biplane views: commissural and } \\
\text { LAX views }\end{array}$ & RAO CRA & $=>$ re-assess perpendicularity \\
\hline 8. & Grasping & $\begin{array}{l}\text { Biplane views: commissural and } \\
\text { LAX views } \\
\text { LAX view (sometimes) }\end{array}$ & RAO CRA & $\begin{array}{l}=>L A X \text { view is of utmost importance } \\
=>\text { adenosine and breath-hold may be necessary } \\
\text { in some cases }\end{array}$ \\
\hline 9. & $\begin{array}{l}\text { Assessment of leaflet } \\
\text { Insertion }\end{array}$ & $\begin{array}{l}\text { Biplane views from commissure } \\
\text { to commissure } \\
\text { 2D LAX } \\
\text { 2D 4-chamber view } \\
\text { SAX transgastric view } \\
\text { 3D en face view } \\
\text { MPR }\end{array}$ & RAO CRA & $=>$ multiple two-dimensional views! \\
\hline 10. & $\begin{array}{l}\text { Procedural Result } \\
\text { (pre and post clip } \\
\text { deployment) }\end{array}$ & $\begin{array}{l}\text { 2D color-Doppler } \\
\text { 3D color-Doppler } \\
\text { MPR Color-Doppler } \\
\text { Pressure gradient } \\
\text { MPR valve area }\end{array}$ & RAO CRA & $\begin{array}{l}\text { => it could be challenging!! REMEMBER: (semi)- } \\
\text { quantitative methods (VC and PISA EROA) have } \\
\text { not been validated in the presence of split MR jets } \\
=>\text { pulmonary vein pattern is a good indicator } \\
\text { => 3D TEE color Doppler could have a role in } \\
\text { quantification } \\
\text { => increase in arterial pressure and LV stroke } \\
\text { volume may also be helpful indicators } \\
=>\text { check trans-mitral gradient and residual MV } \\
\text { area } \\
\text { => careful evaluation of complications (e.g., } \\
\text { significant IAS shunt, pericardial effusion) }\end{array}$ \\
\hline 11. & Clip Deployment & Biplane views & RAO CRA & \\
\hline 12. & System Removal & $\begin{array}{l}\text { Multiple 2D ME views } \\
\text { 3D overhead view }\end{array}$ & RAO CRA & \\
\hline
\end{tabular}

SAX: short axis; AP: antero-posterior; LAO: left anterior oblique; RAO: right anterior oblique; IAS: interatrial septum; PFO: patent foramen ovalis; LAX: Iong axis; LA: left atrium; SGC: steerable guide catheter; CRA: cranial; ME: mid esophageal; MV: mitral valve; MPR: multiplanar reconstruction; VC: vena contracta; EROA PISA: effective regurgitant orifice area with proximal isovelocity hemispheric surface area; MR: mitral regurgitation; TEE: transesophageal echocardiography

could be acceptable ( 3.5-4.0 cm above the annular plane) as coaptation is dislocated deeper in the left ventricle. Moreover, slight differences in height above the annulus could be determined by the planned positioning of the device in terms of a lateral $v s$. medial regurgitant lesion. A lower TSP site is required for a lateral defect, while higher TSP site is required for a medial defect as a low TSP will move the clip below the mitral annulus when deflecting the system toward the mitral annular plane from lateral to medial. 

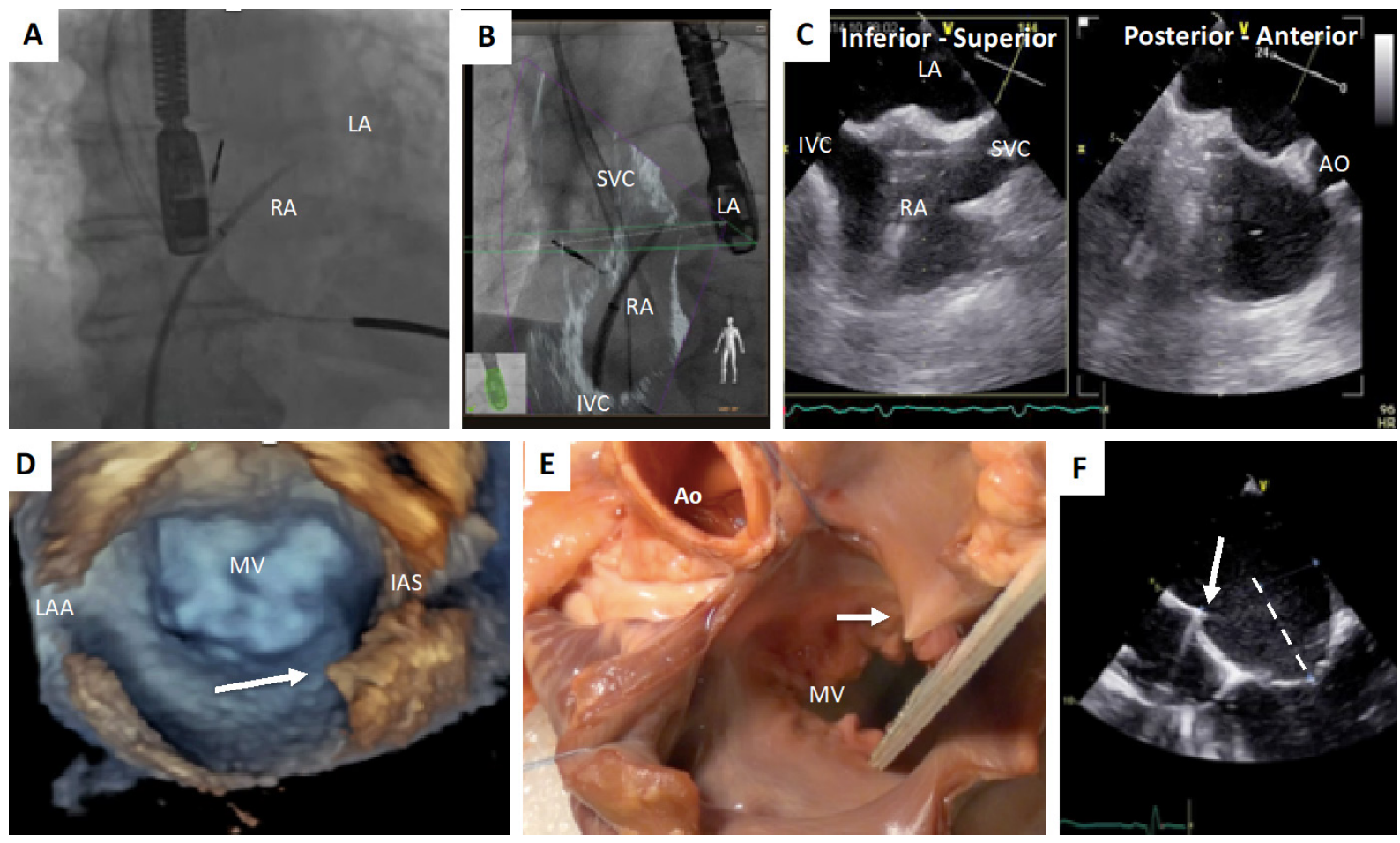

Figure 1. Transseptal puncture. A: AP fluoroscopic projection; B: fusion imaging: LAO fluoroscopic projection with superimposition of the corresponding TEE view (2D bicaval view); C: biplane imaging: the most used views for TSP guidance: bicaval view (left panel) and SAX-B view (right panel). A sharp tenting should be clearly visualized and a superior and posterior localization; D: 3D overhead perspective of the LA clearly highlights the tenting (white arrow); E: TSP simulation on anatomical specimen (pig heart): MV and Ao are clearly visible, while a withe arrow highlights the tenting on the left side of the IAS; F: ME 4-chamber view is used to measure the height between the tenting (puncture site, white arrow) and the annular plane. AP: antero-posterior; RA: right atrium; LA: left atrium; IVC: inferior vena cava; SVC: superior vena cava; Ao: aortic root/valve; MV: mitral valve; IAS: interatrial septum; LAA: left atrial appendage; LAO: left anterior oblique; TEE: transesophageal echocardiography; TSP: trans-septal puncture; SAX: short axis

With atrial dilation which is common in MR patients, the location/angle of the interatrial septum (IAS) in relation to the MV plane may be distorted. This distortion is difficult to appreciate on $2 \mathrm{D}$ imaging; thus, $3 \mathrm{D}$ TEE confirmation of TSP location is recommended in such cases. Access via a patent foramen ovalis is not recommended, although the entry site into the LA would be superior as the defect is generally too anterior and the tunnel constrains the trajectory of the steerable guide catheter (SGC) tangent to the IAS and toward aortic root. Access via an atrial septal defect (ASD) is also not recommended for two main reasons: (1) the size of the defect generally does not match the size of the SGC; and (2) in most cases the septum does not provide proper support for a stable position of the SGC and there is an increased risk of septal rupture.

\section{Steerable guide catheter insertion into left atrium}

Crossing of the IAS should be visualized in the 2D SAX-B view or intermediate view between SAX and bicaval views or by several $3 \mathrm{D}$ perspective of LA. Both views allow visualization of the distal portion of the transseptal needle and its passage into the LA [Figure 2]. The insertion of the SGC should be carefully monitored by $2 \mathrm{D}$ (mid-esophageal short-axis, long-axis, and four-chamber views are recommended), 3D overhead perspective of LA and fluoroscopic imaging, in order to avoid injuries of the LA wall. Persistence of tenting denotes that the SGC has still not completely crossed the septum.

The dilator should be removed when the SGC is at least $2 \mathrm{~cm}$ across the IAS. Fluoroscopy and echocardiography both help in differentiating the dilator from the SGC. 

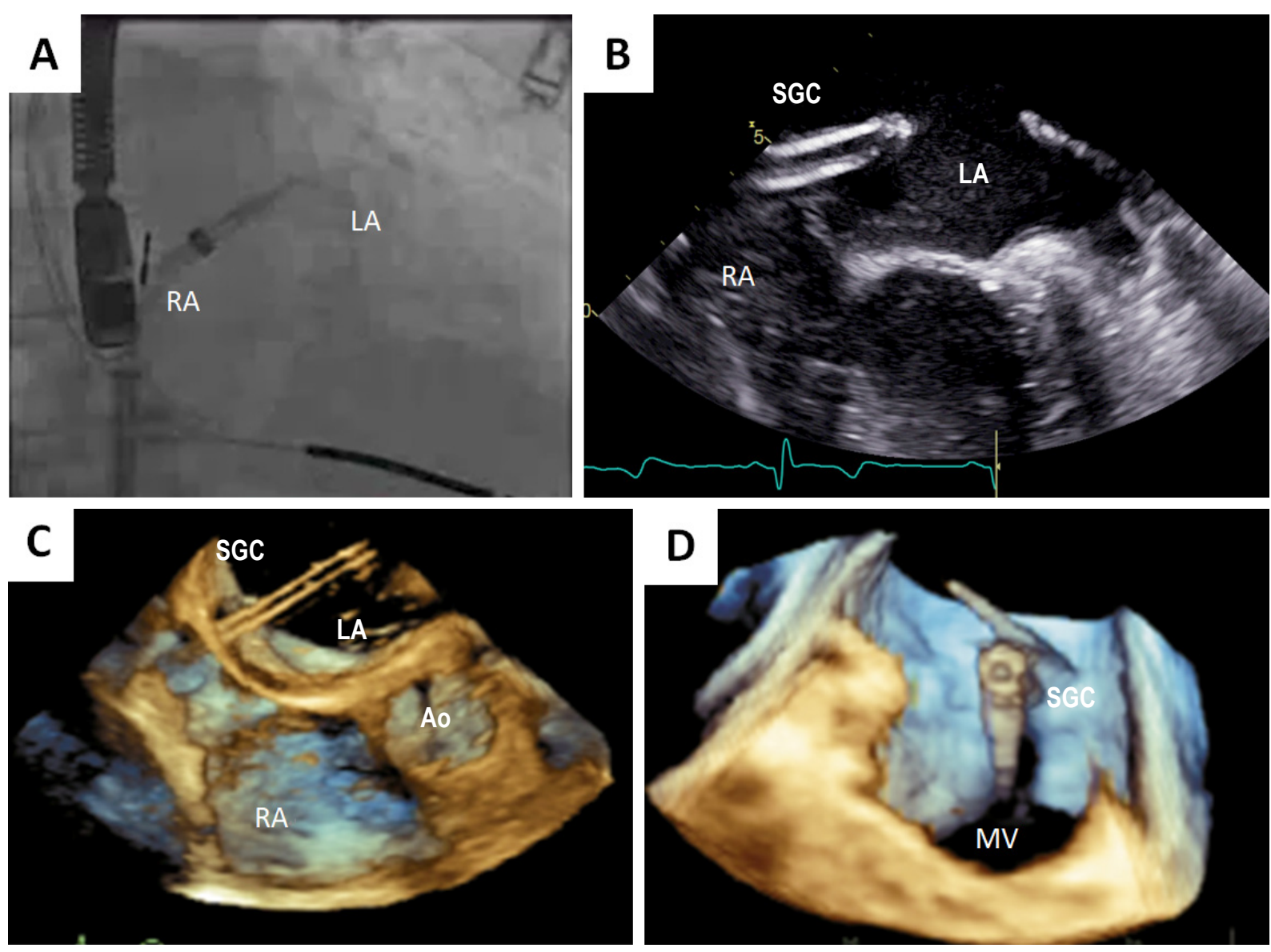

Figure 2. Steerable guide catheter insertion into left atrium. A: AP fluoroscopic projection showing both transseptal sheath and dilator inside the LA; B-D: SGC trough the septum inside the LA after removing the dilator: the dilator can be identified by its typical echogenic coils striations at the cone tip, whereas the tip of the SGC is marked with a radiopaque echo bright rail-road shaped artifact and can be identified by 2D TEE (B) and 3D TEE (C: lateral perspective; D: en face perspective). SGC: steerable guide catheter; RA: right atrium; LA: left atrium; Ao: aortic root/valve; MV: mitral valve

Clip delivery system advancement through the catheter into left atrium

The clip delivery system (CDS) is then gently advanced into the LA through the SGC under fluoroscopic and TEE guidance [Figure 3]. The 3D TEE overhead perspective of the LA offers the best comprehensive view of spatial relationships among structures and device, as gross DS movements in the center of the LA are required. When the DS is directly adjacent to the lateral atrial wall, 2D imaging (short axis and 4 chamber views, or simultaneous multiplane view) is more useful thanks to the better spatial resolution for evaluating the relationship between the Clip and LA structures and it is the preferred imaging modality for guiding the steering toward MV plane. At this stage, the 2D TEE view (usually in between short axis and bicaval views) is also useful to confirm the position of SGC across the IAS inside the LA.

\section{Steering and positioning the clip in the left atrium}

The DS is steered towards the MV over the target lesion (the tip of the clip should point towards the largest color flow convergence zone and should split the regurgitant jet) [Figure 4]. A series of steering maneuvers in anterior-posterior and/or medial-lateral directions allow the achievement of the desired position over the MV target lesion. This step is usually monitored by $3 \mathrm{D}$ overhead perspective of the LA for gross DS movement and $2 \mathrm{D}$ simultaneous biplane views for fine adjustments: a mid-esophageal commissural view $\left(\sim 60^{\circ}\right)$ to perform medial-lateral adjustments and a long-axis view at $120^{\circ}-150^{\circ}$ (LAX view) to monitor anterior-posterior adjustments. 


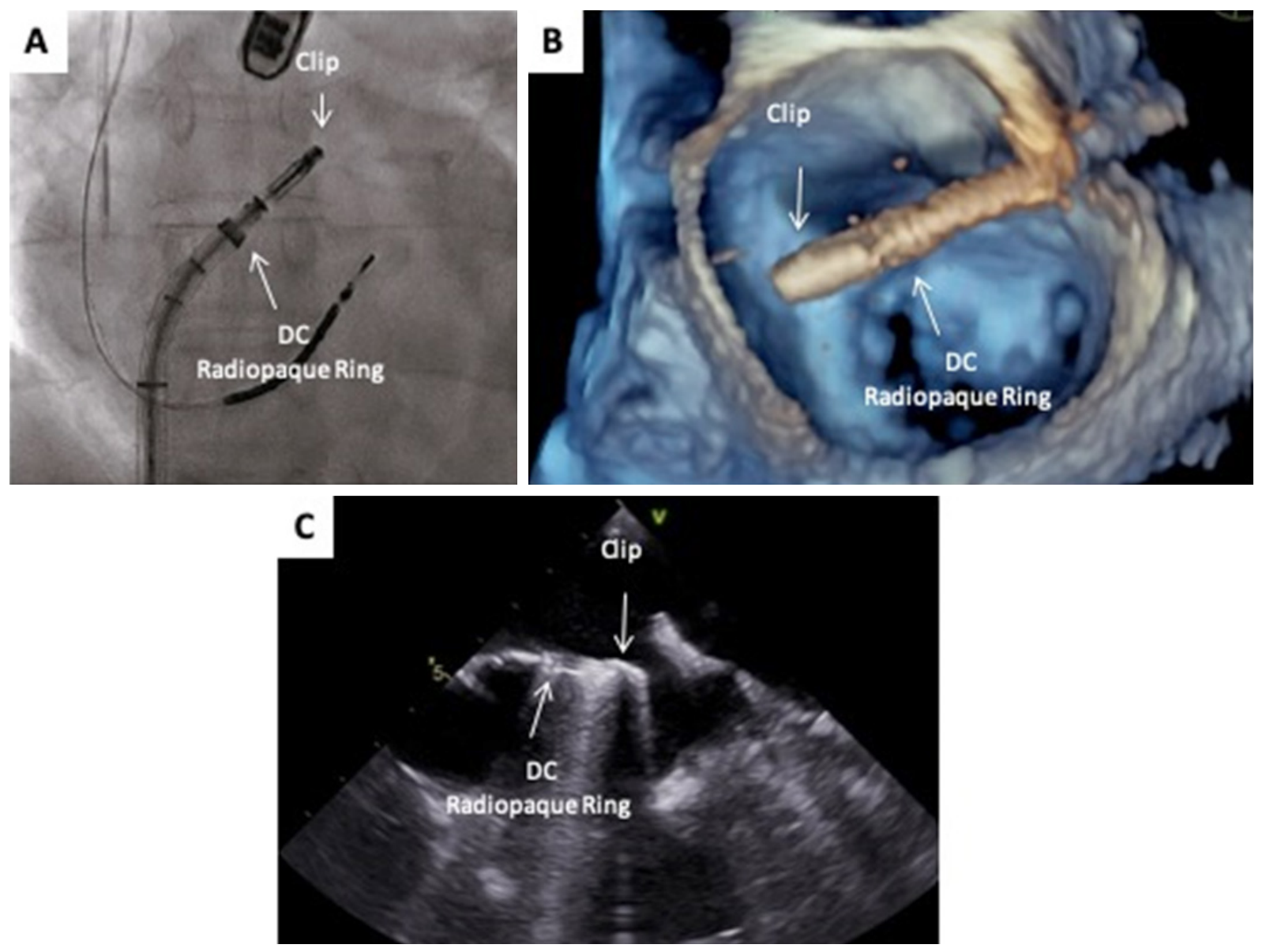

Figure 3. Clip delivery system advancement through the catheter into left atrium. The clip delivering system inside LA imaged with different modalities, A: AP fluoroscopic view; B: 3D overhead perspective of the LA; C: 2D mid-esophageal 4 chamber view. DC: clip delivery catheter; AP: antero-posterior; LA: left atrium

Axial alignment of the clip delivery system

The proper trajectory should ensure perpendicularity of the device in relation to the MV plane, avoiding a slanting one. Misalignment can lead to incorrect advancement of DS into LV, affecting both symmetry and efficacy of the grasping (e.g., difficult or insufficient grasping of one of the leaflets, distortion of the coaptation line). This aspect is of utmost importance in cases of commissural lesions to avoid chordal entrapment. Fluoroscopy, echocardiography and fusion imaging guidance are useful for this procedural step [Figure 5].

Alignment of the clip arms to the coaptation line

Once the appropriate axial alignment of the DS is achieved over the target lesion, the arms of the Clip can be deployed. The Clip arms should be oriented perpendicularly to the coaptation line. Clip orientation is monitored by $3 \mathrm{D}$ TEE en face view of the MV together with $2 \mathrm{D}$ simultaneous biplane views [Figure 6]. In the case of central Clip location, if proper position is achieved, no Clip arms should be seen in the commissural view and both clip arms should be visualized in full length in the LAX view. Additionally, in the RAO cranial fluoroscopic projection, clip arms should not be visible. Instead, if the Clip is positioned in the lateral or medial region of the valve, Clip arms can be partially visible in the commissural view. In this last case, the LVOT view can be useful for assessment of fully opened Clip arms. Additionally, short-axis transgastric view can be used to confirm perpendicularity of Clip arms to the coaptation line. 

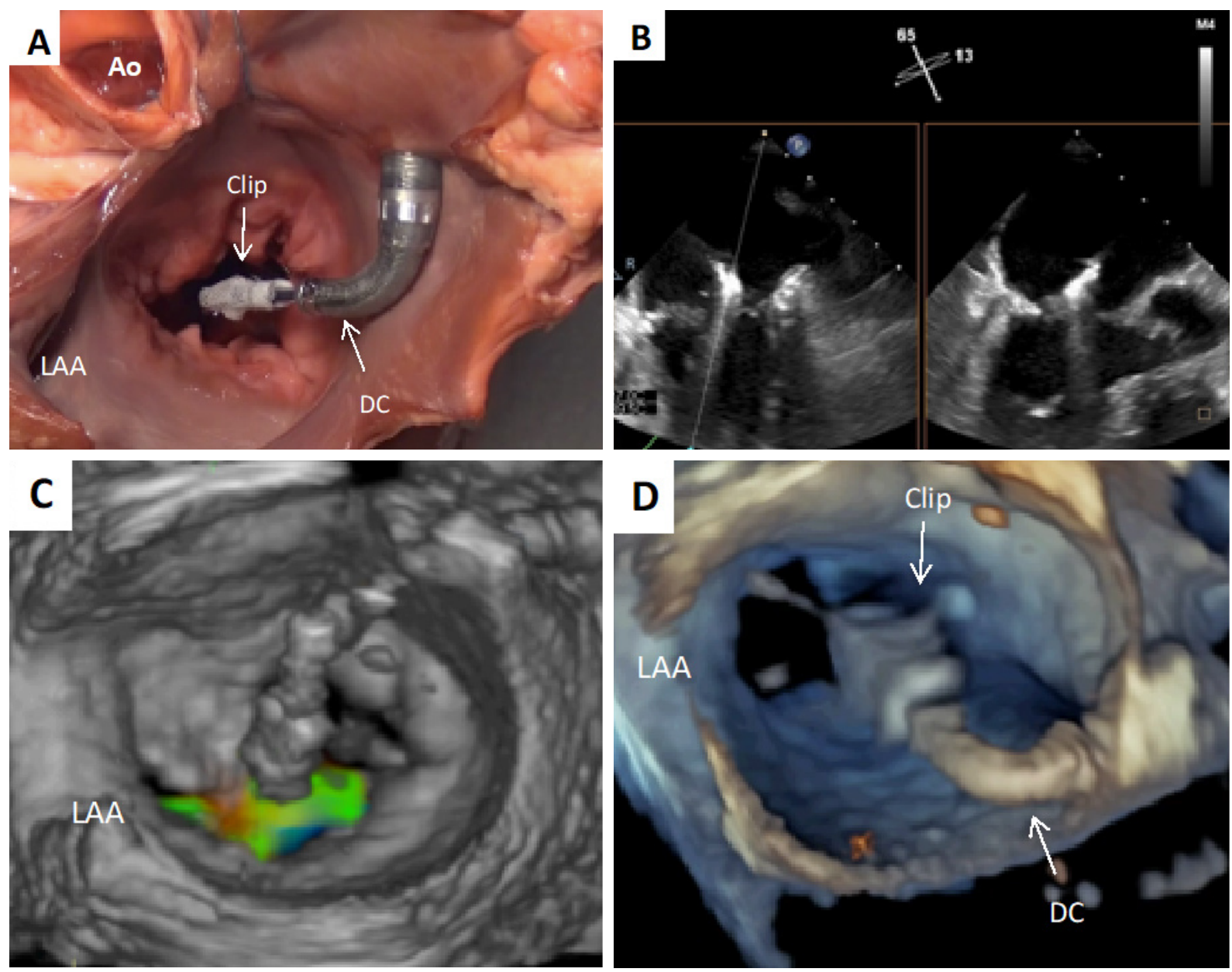

Figure 4. Steering and positioning the clip in the left atrium. A: anatomical specimen (pig heart) showing the steering of the clip towards the MV: reference landmarks are LAA and Ao; B: biplane imaging guiding the steering towards the MV: commissural view for mediolateral and LAX view for postero-anterior guidance; C, D: 3D overhead perspective of the LA with (C) and without (D) color guiding the steering and the positioning of the device. DC: Clip delivery catheter; LAA: left atrial appendage; LA: left atrium; Ao: aortic root/valve; MV: mitral valve; LAX: long axis

\section{Advancement into left ventricle}

The DS is then advanced distally across the MV, with Clip arms partially closed $\left(60^{\circ}\right)$, approximately $2 \mathrm{~cm}$ below the MV into the LV under fluoroscopic and TEE guidance. This step is monitored by $2 \mathrm{D}$ simultaneous biplane views (commissural and LAX views) [Figure 7]. Once the clip is below the MV plane, and the arms are fully opened, correct positioning should be verified by the visualization of mitral leaflets moving freely above Clip arms and splitting of the MR jet. In order to preserve perpendicularity with the coaptation line, it is important to reconfirm the correct Clip orientation under simultaneous biplane views and $3 \mathrm{D}$ en face view, as the Clip may rotate during the passage across the MV. Using the $3 \mathrm{D}$ en face view, it is possible to progressively reduce the gain until the MV leaflets become almost transparent, allowing visualization of the Clip arms proper orientation, or alternatively using the simultaneous $3 \mathrm{D}$ display from LA and LV. Inside the LV, changes in Clip arms orientation should be minimal to avoid entanglement in the chordae tendinae. In case of significant orientation adjustment ( $>90^{\circ}$ in each direction), the Clip should be everted and withdrawn back into the LA where its orientation may be safely manipulated, avoiding chordae entanglement that may make difficult or even impossible to remove the Clip or even damage MV. 

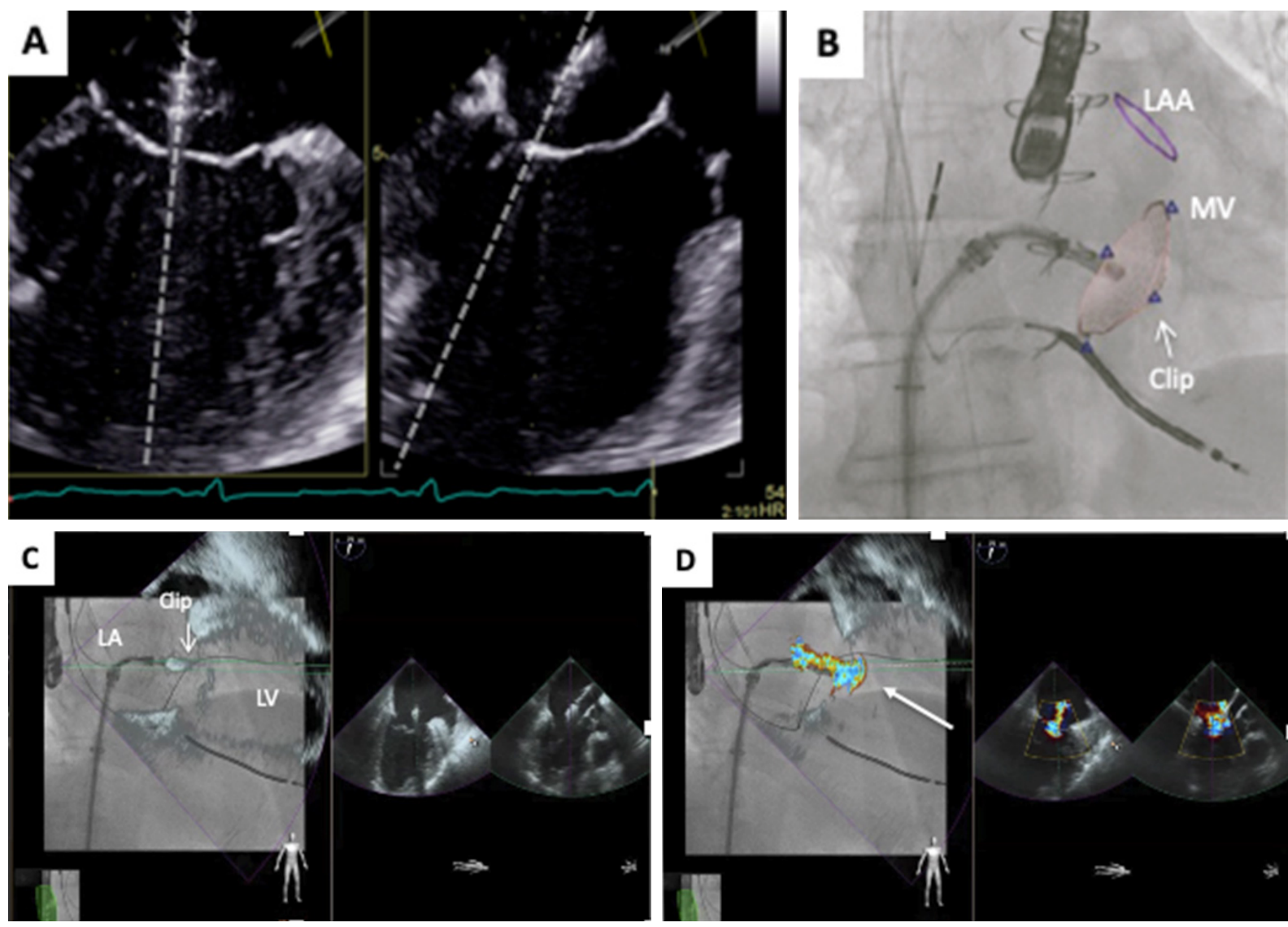

Figure 5. Axial alignment of the clip delivery system. A: biplane imaging, starting from the commissural view as the main view and the LAX view as the derived one, allows for medial-lateral and anterior-posterior clip adjustments. The perpendicularity of the system with respect to the MV plane should be achieved in both views; B-D: fusion imaging showing the same procedural step: RAO CRA fluoroscopic projection with superimposed commissural view with (D) and without (C) color. LAA: left atrial appendage; LV: left ventricle; LA: left atrium; MV: mitral valve

\section{Leaflets grasping}

Once the Mitraclip ${ }^{\mathrm{TM}}$ is in the proper position in the left ventricle, it is useful to recheck its orientation; in addition, 3D lateral perspective of the left atrium and ventricle could be useful to evaluate the angle of the clip before full grasping.

Subsequently, leaflet grasping is performed by slowly retracting the system back towards the LA, to allow the leaflets to come to rest on the Clip arms. Once both leaflets are visualized over the Clip arms with tips ideally adjacent to the shaft, the grippers are lowered onto the leaflets. This step is usually monitored by a $2 \mathrm{D}$ simultaneous biplane view, focused on LAX view, in addition to fluoroscopy [Figure 8]. In cases of paracommissural Clip placement, simultaneous biplane view starting from the commissural view as a reference plane may not provide adequate visualization of equal Clip arm lengths together with the anterior and posterior leaflets on the derived LAX view (due to inadequate angulation of the elevation plane). It could be useful to transiently refer to the $2 \mathrm{D}$ LAX view which provides more adequate visualization of equal Clip arm full lengths.

It is important to continuously visualize leaflet insertion while grasping to avoid rolling leaflets/chordae. Partial closure of the Clip until the arms angle is $\sim 60^{\circ}$ is recommended, and this distinct "V" shape should be maintained on fluoroscopy. When the Clip appears properly positioned, leaflet insertion and MR reduction appear satisfactory without inducing stenosis, the Clip can be fully closed. 

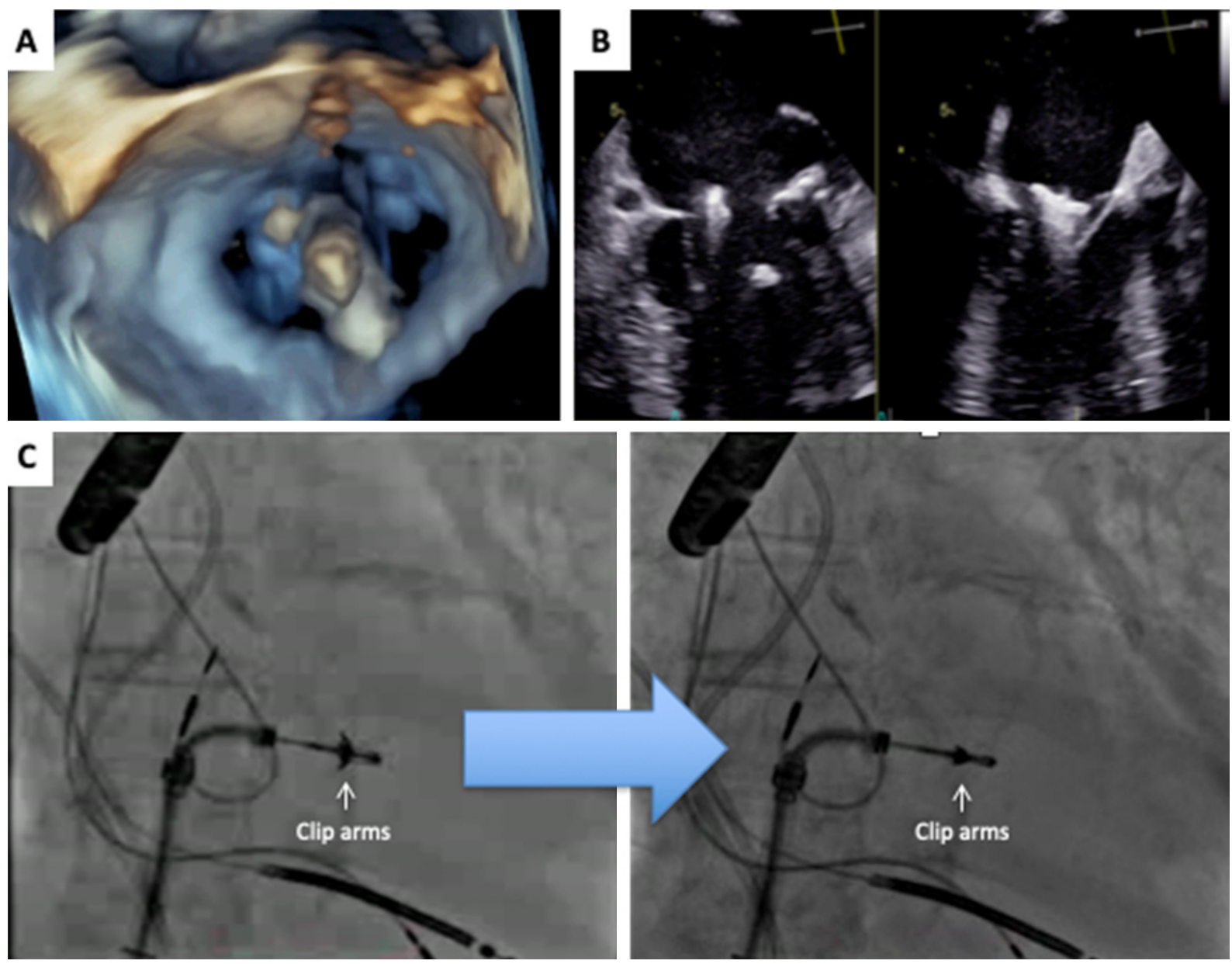

Figure 6. Alignment of the clip arms to the coaptation line. A: 3D MV en-face view guiding clip arms orientation with respect to the coaptation line; B: biplane imaging: clip arms should be visible only in LAX view; C: RAO CRA fluoroscopic view: after adequate rotation according to the echo guidance, clip arms are not visible anymore (right panel). LAX: Iong axis view; RAO CRA: right anterior oblique cranial projection; MV: mitral valve

\section{Assessment of leaflet insertion}

The acquisition of a long loop is helpful as the grasping can be re-evaluated whenever needed. Adequate leaflet insertion is verified when direct and indirect signs are simultaneously present. Direct signs [Figure 9]: (1) The length of the leaflet captured inside the Clip should be $\geq 5 \mathrm{~mm}$ (some have reported at least $4 \mathrm{~mm}$ ), with both leaflets inserted into the atrial aspect of the closed Clip arms (the length of leaflet captured inside the Clip is determined by subtracting the leaflet length outside the Clip from the corresponding leaflet length at baseline);

(2) The leaflet draped over the closed arms should have a reduced mobility relative to the tips of the Clip arms. Leaflets motility can be easily assessed by the $2 \mathrm{D}$ simultaneous biplane view, using the commissural view as the main view and moving the elevation plane along the MV from the posterior-medial orifice to the anterior-lateral one, closely to the edge of the Clip. The quality of insertion of the posterior and anterior leaflets is usually best evaluated in LAX and 4-chamber view respectively;

(3) The occurrence of a double MV orifice: the 3D en face view of MV from LA or LV perspectives as well as the $2 \mathrm{D}$ short axis transgastric view of MV are helpful to assess the new geometry of the valve and should show a double-orifice valve with an adequate tissue bridge over the Clip arms. In addition, multiplanar reconstruction of the same $3 \mathrm{D}$ dataset allows for further evaluation of the leaflets. 

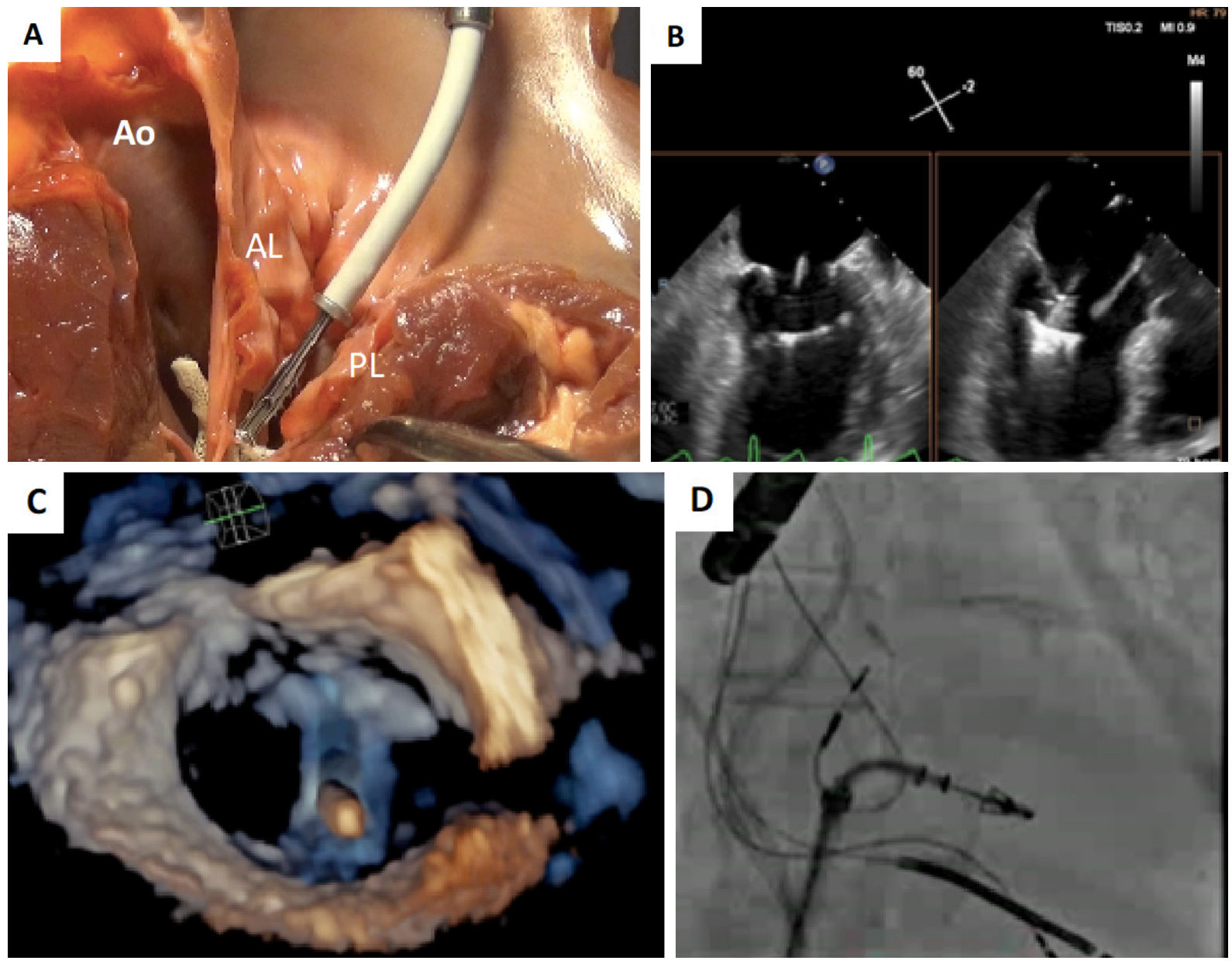

Figure 7. Advancement into LV. A: anatomical specimen (pig heart) showing clip advancement into LV: lateral perspective of the clip advanced into the left ventricle; B: Bi-plane imaging showing clip advancement into left ventricle; C: 3D MV en face view after reducing the gains shows clip arms and their orientation across MV; D: RAO fluoroscopic view shows clip inside LV across the MV. Ao: aortic valve/root; AL: anterior leaflet, PL: posterior leaflet; RAO: right anterior oblique; LV: left ventricle; MV: mitral valve
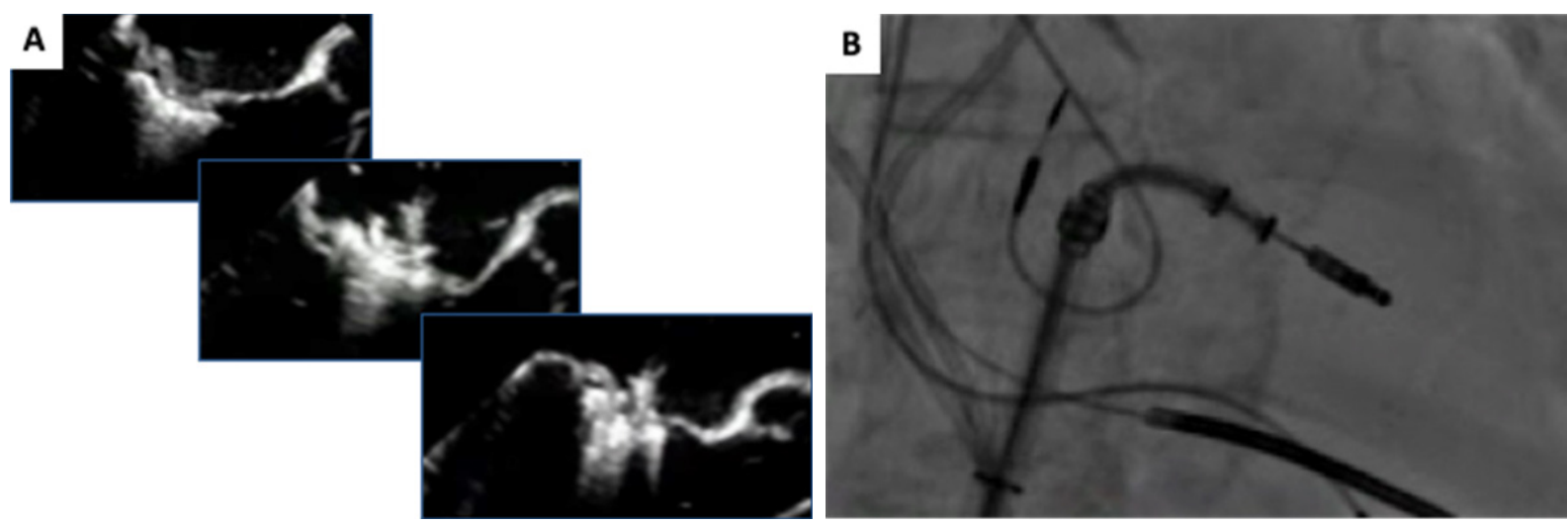

Figure 8. Leaflets Grasping. A: sequential zoomed LAX view of leaflets grasping; B: RAO fluoroscopic projection showing clip with closed arms. LAX: long axis view; RAO: right anterior oblique

Indirect signs of adequate leaflets grasping are [Figure 10]: the presence of MR reduction, the absence of intraclip jet (that may suggest inadequate amount of leaflet tissue grasped into Clip arms), the appearance of spontaneous echo contrast in LA/LAA. 

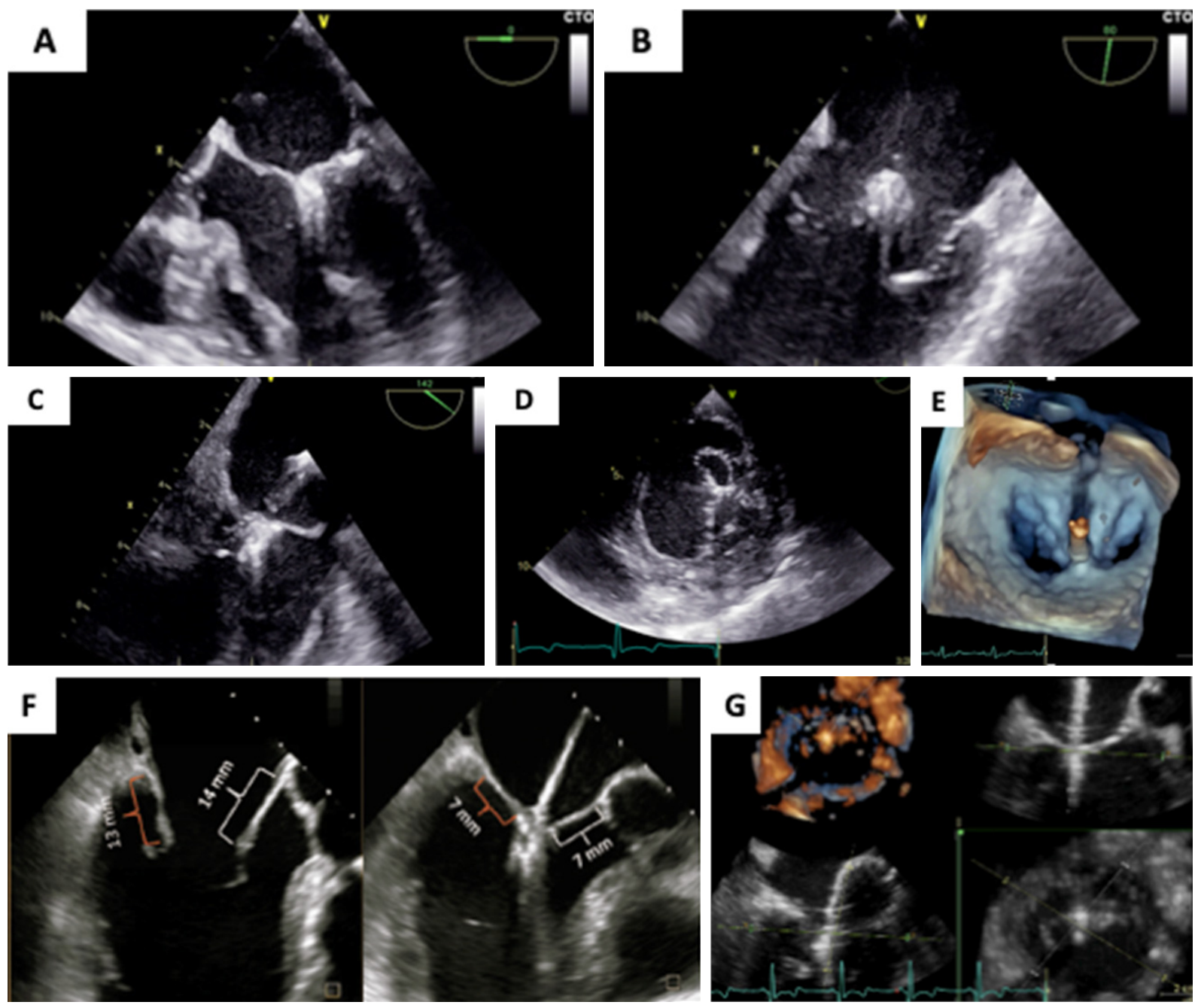

Figure 9. Assessment of leaflets insertion. Verification of satisfactory grasp of the leaflets requires multiple views: 4-chamber view (A), commissural view (B), long axis view (C), transgastric short axis view (D), 3D en face view (E); measurement of leaflets length before and after grasping (F); multiplanar reconstruction allows simultaneous visualization of different views (G)
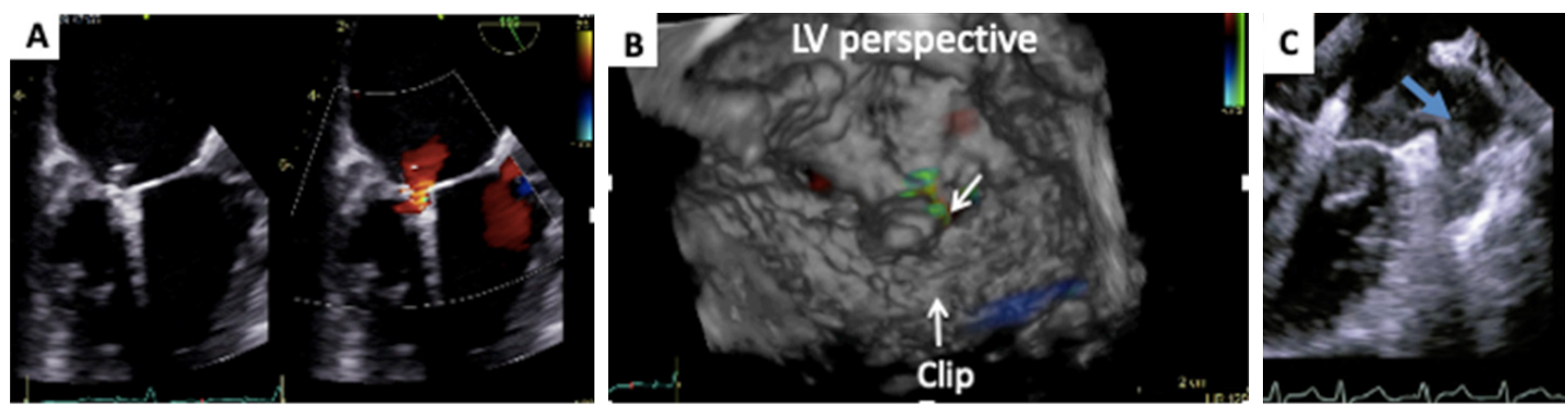

Figure 10. Assessment of Leaflets Insertion: Indirect Signs. 2D color Doppler (A) and 3D LV perspective (B) showing a residual intraclip jet; appearance of spontaneous echocontrast after leaflet grasping (C). LV: left ventricle

Assessment of result before Clip release

The best result should be a proper balance between tolerable transmitral pressure gradient (TMPG) and adequate reduction of MR. 


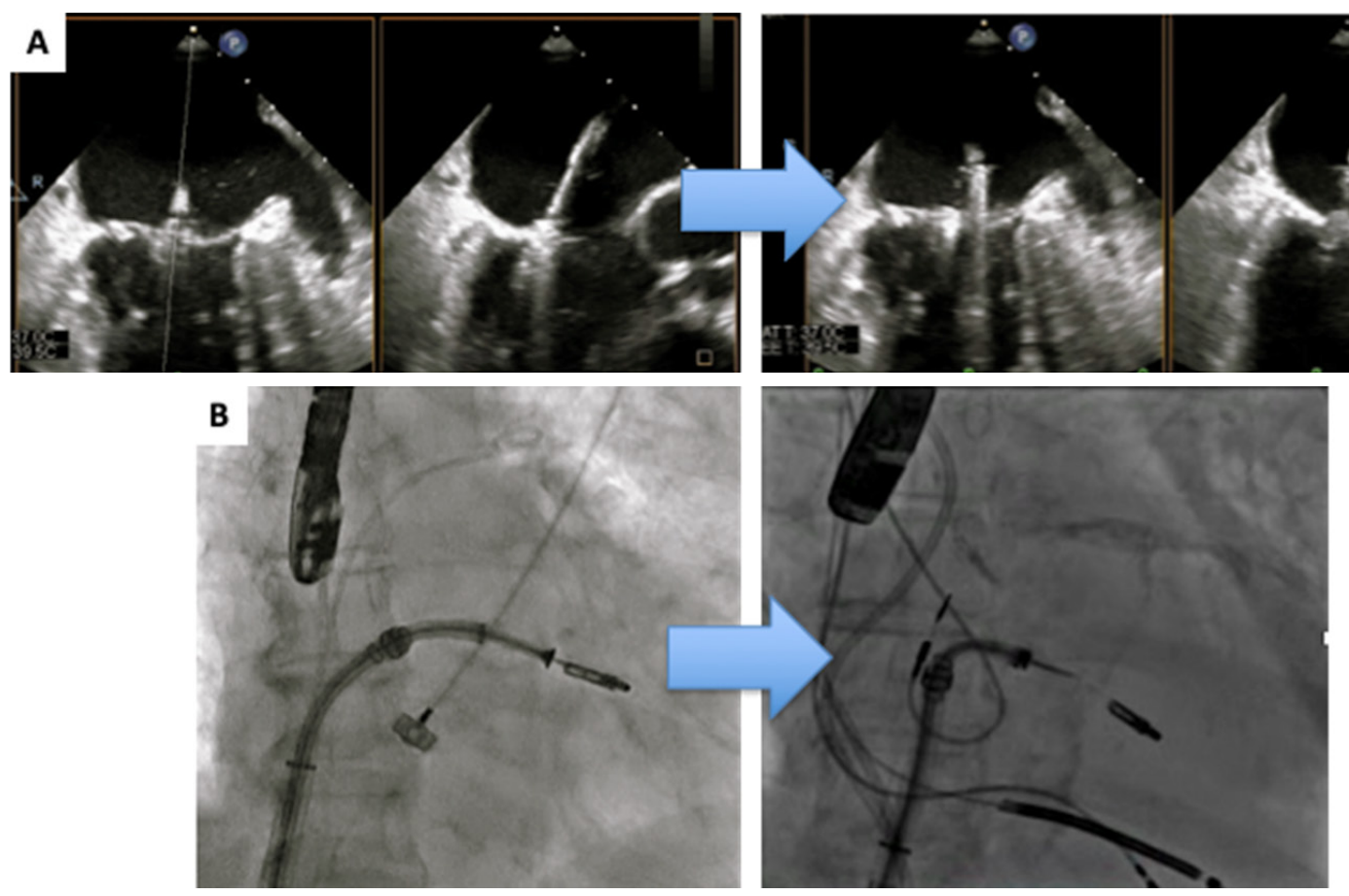

Figure 11. Clip release. Biplane imaging (A) and right anterior oblique fluoroscopic projections (B) showing clip release

The risk of mitral stenosis has to be evaluated by the assessment of diastolic TMPG via continuous-wave (CW) Doppler after the placement of each Clip.

Planimetric assessment of the MV area provides an additive information. It should be preferably assessed by using 3D imaging, which allows for multiplanar reconstruction ${ }^{[8]}$. Alternatively, 2D planimetry could be performed in the mid-diastole phase using the transgastric short-axis view. In both cases, the edges of the MV leaflets should be clearly visible, allowing the inner edge of each orifice to be traced and the areas added to calculate the total size of the newly formed orifices.

By combining both information, TMPG and MVA, it is possible to estimate the risk of iatrogenic mitral stenosis more accurately. An MVA $\leq 1.5 \mathrm{~cm}^{2}$ and a TMPG $\geq 5 \mathrm{mmHg}$ were considered criteria to indicate significant MS in the EVEREST studies ${ }^{[9,10]}$.

Moreover, intraprocedural TMPG measured by TEE under general anesthesia conditions potentially underestimates the hemodynamic impact of reduced MVA in daily life with exercise, which operators should be aware of when deciding on implanting one or more clips ${ }^{[10]}$.

Together with MVA and TMPG, the assessment of the final geometry of the MV should ensure: (1) each clip is placed symmetrically on both leaflets and that the Clip is not biased towards one of them; and (2) excessive distortion of the leaflets is avoided as it may lead to unbalanced traction and potentially cause partial Clip detachment or leaflet rupture during follow-up. 3D en face view with atrial or ventricular perspective is a fundamental imaging tool for this evaluation.

\section{Clip release}

Once the Clip position is appropriate and MR effectively reduced, the Clip is detached from the catheter shaft usually under 2D imaging and fluoroscopic guidance [Figure 11]. A stable Clip position has to be reconfirmed and the grade of residual MR should be reassessed by Color Doppler, as minor changes can occur when the tension transferred via the DS disappears. 

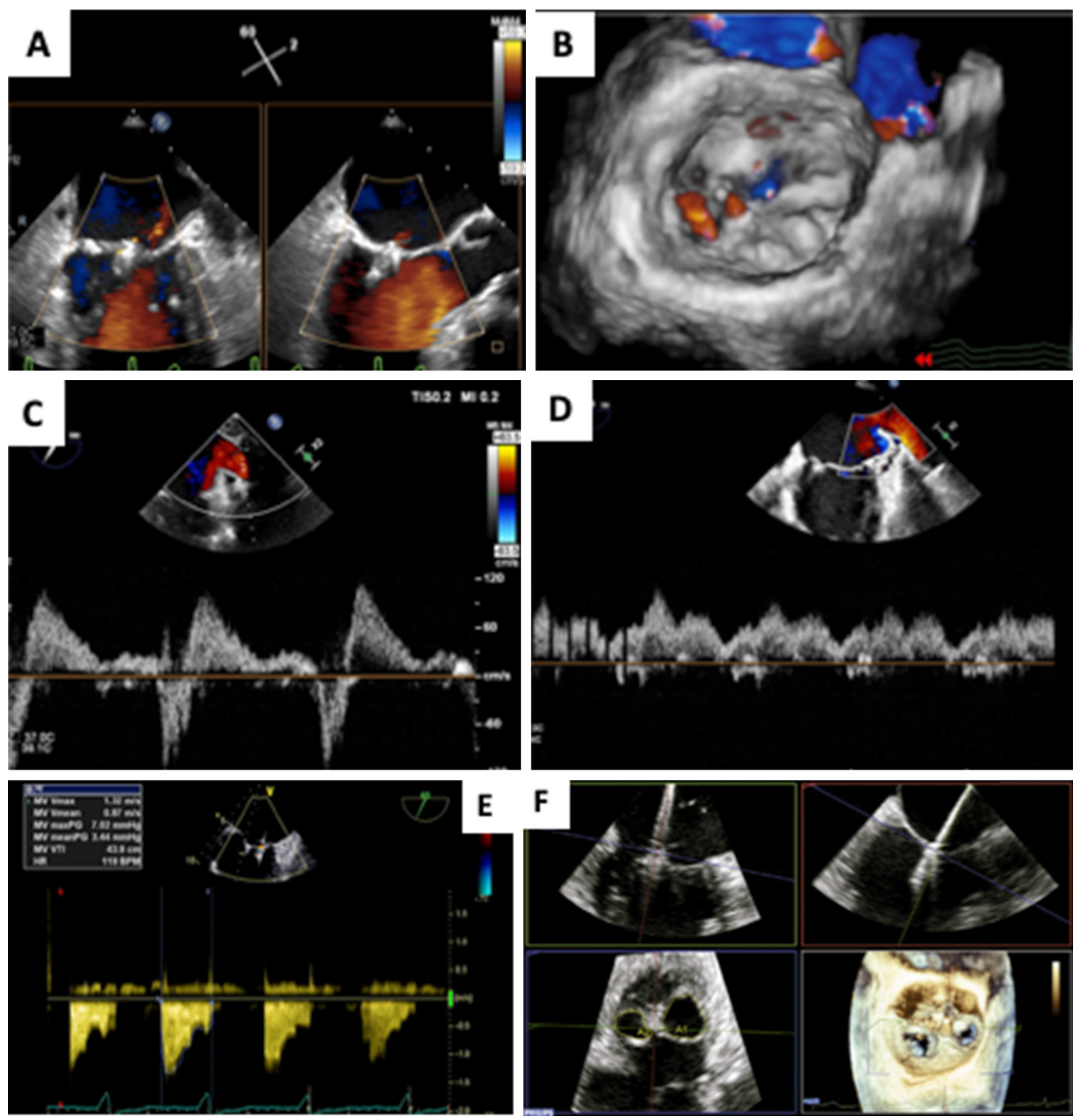

Figure 12. Assessment of result after clip release. A: biplane imaging and $3 D$ en face (B) with color Doppler showing a residual jet from the lateral orifice; C: baseline evaluation: systolic flow reversal at Pulse Wave Doppler interrogation of the LSPV; E: post-procedural evaluation: normalization of systolic flow in the LSPV; F: evaluation of the transmitral pressure gradient; G: Multiplanar reconstruction: evaluation of residual mitral valve area. LSPV: left superior pulmonary vein

Assessment of result after Clip release

Similar hemodynamic conditions and the same ultrasound settings are required to make a valuable comparison between baseline and post-Clip MR. In particular, blood pressure needs to be normalized.

A multi-modal approach provides the most suitable and appropriate method to characterize and quantify residual $\mathrm{MR}^{[11]}$ [Figure 12]:

(1) Color-Doppler is the main initial modality for MR assessment, in terms of site, number of jets, eccentricity, vena contracta, and flow convergence, throughout the whole procedure, allowing rapid and easy evaluation of MR. PISA method is less reliable and therefore not recommended for flow quantitation and EROA calculation; 
(2) The 3D color-Doppler vena contracta area (VCA) seems promising as it is able to overcome the limits and the assumption of EROA-PISA evaluation ${ }^{[12,13]}$. Indeed, each jet that is deemed significant would need to be separately analyzed for VCA, since they are often in different planes and with different orientations, and their VCAs can be added. It must be remembered that 3D color Doppler could be limited by a low frame rate. Furthermore, 3D color can help identify, assess, and localize residual eccentric or wall-hugging MR jets not seen on $2 \mathrm{D}$ color flow imaging. However, to date there is no sufficient data to routinely recommend 3D VCA as a strong parameter to quantify residual MR after clip placement;

(3) The pulmonary vein flow pattern is very useful to assess residual MR grading. Normalization of pulmonary vein flow after MV interventions strongly suggests that MR has been reduced to mild;

(4) Invasive hemodynamic parameters, such as the resolution of regurgitant atrial $v$ wave and reduction of left atrial or pulmonary pressures, provide important additional clues to improvement in MR severity. It is also common to observe an increase in systolic blood pressure immediately after a successful reduction in severe MR, reflecting an increase in forward stroke volume;

(5) The mitral inflow velocity pattern (decrease in mitral E velocity and velocity time integral) may be helpful in assessing reduction of $\mathrm{MR}$, as a change from an E-wave-dominant to an A-wave dominant pattern could suggests mild residual MR. A diastolic TMPG rise, without significant reduction of MVA could be an indirect sign of residual moderate or greater MR. In such a setting, an additional Clip may "paradoxically" reduce the diastolic TMPG by the improvement of MR;

(6) Appearance of spontaneous echocontrast in the LA after MV repair also suggests significant reduction in MR severity;

(7) Left ventricular outflow tract velocity integral as assessed in deep transgastric views may be helpful in demonstrating an increase in forward systemic flow;

(8) A decline in LV ejection fraction after MitraClip ${ }^{\mathrm{TM}}$ procedure also could suggest significant MR reduction and thus increased afterload.

\section{System removal}

After release of the Clip, the CDS is withdrawn into SGC. The distal end of the DS, also called the atraumatic tip, may injure LA structures during withdrawal into the SGC. This maneuver is usually monitored by multiple views in $2 \mathrm{D}$ imaging. At this stage, an intermediary between short axis and bicaval views, is also useful to confirm that the SGC is maintained sufficiently into the LA, as a second Clip implantation may be required. If no additional Clip is needed, the SGC is withdrawn back across the IAS and out of the femoral vein access.

\section{Atrial septal defect evaluation}

After system removal, the residual shunt and size of iatrogenic IAS shunt should be evaluated. Assuming no additional damage to the septum and predominantly left to right flow, the defect is generally of no clinical significance ${ }^{[14]}$.

\section{Complications}

Percutaneous edge to edge MV repair is generally a safe procedure with good hemodynamic tolerance even in high-risk patients and is associated with few major complications ${ }^{[15,16]}$. Serious complications may occur at a low rate and can be promptly identified by echocardiographic monitoring during the procedure. Potential complications may be represented by:

(1) The occurrence of thrombus on intracardiac devices, such as guidewires and/or delivery sheaths;

(2) Acute severe hypotension caused by cardiac tamponade, acute decline of LV function, or worsening of MR;

(3) Pericardial effusion and cardiac tamponade due to perforation of the LV free wall or aortic puncture during TSP;

(4) MR worsening, subtended by three major mechanisms: leaflet or chordal damage; loss of leaflet 

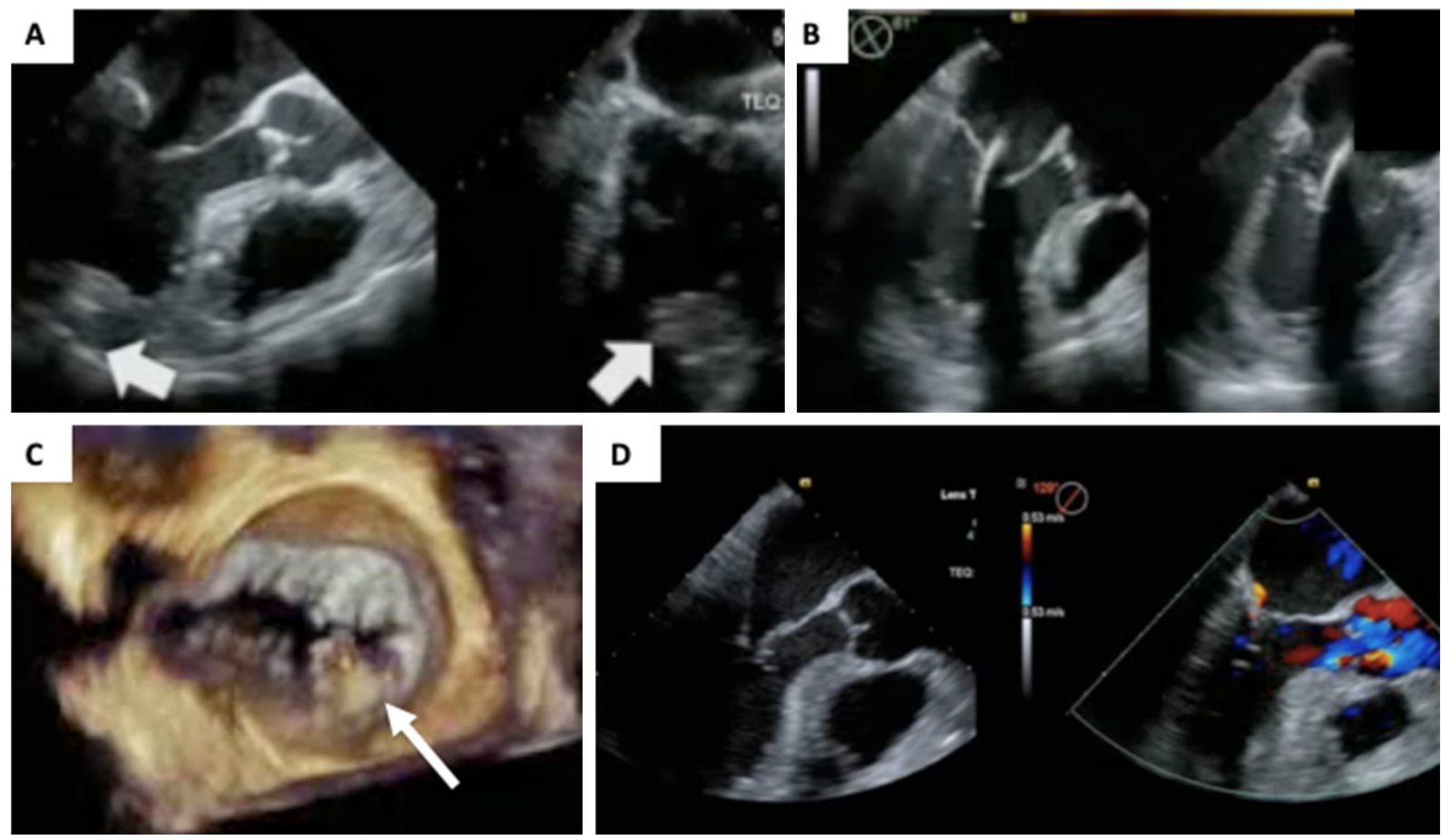

Figure 13. Procedural guidance of the transapical beating heart chordal implantation. A: identification of the LV apex through "finger testing": the interventionist pushes the apex (white arrow) and the imager checks its position on biplane imaging; B: the system is directed towards the LA on simultaneous biplane LAX and commissural views, avoiding entrapment into subvalvular apparatus. After entering the $L A$, echocardiographic imaging is switched to 3D surgical view (C), targeting the prolapsing segment (white arrow). After confirmation of leaflet grasping and capture, the device is pulled out from the LV apex and tension is adjusted, until effective MR reduction is shown with color Doppler interrogation (D). LV: left ventricular; LA: left atrium; LAX: long axis; MR: mitral regurgitation

insertion and partial clip detachment, also referred as single leaflet device attachment, and may occur in the case of insufficient leaflet grasping. Depending on the underlying cause, acute MR may require emergency circulatory support and/or bail-out MV surgery;

(5) Iatrogenic mitral stenosis.

\section{CHORDAL APPROACH: TRANSAPICAL BEATING HEART CHORDAL IMPLANTATION}

This method applies to all the basic steps of the conventional surgery in which delivery and adjustment of chordal length after implantation is done on the beating heart without the use of the cardiopulmonary bypass.

The most suitable lesion for this approach is an isolated P2 segment flail or with a minimum overriding of at least $9 \mathrm{~mm}$, without significant annular dilatation and severe LV dilatation with leaflets tethering.

Under general anaesthesia a mini-thoracotomy transapical approach is performed under TEE guidance [Figure 13]: the polytetrafluorethylene (ePTFE) chords are delivered to the leaflets and then subsequently adjusted to optimize MR reduction. Two currently available devices are NeoChord DS1000 system (NeoChord, Inc., Eden Praire, MN) ${ }^{[17-19]}$ and Harpoon (Edwards Lifescience, Irvine, USA $)^{[20]}$.

After a standard left lateral mini-thoracotomy in the fifth intercostal space to access the LV apex, the system is directed towards the LA on 2D-TEE guidance (simultaneous multiplane LAX + commissural views) avoiding native subvalvular apparatus entrapment and staying in the central part of the MV (A2P2 segments). After trans-mitral navigation and entering the LA, echocardiographic imaging is switched 


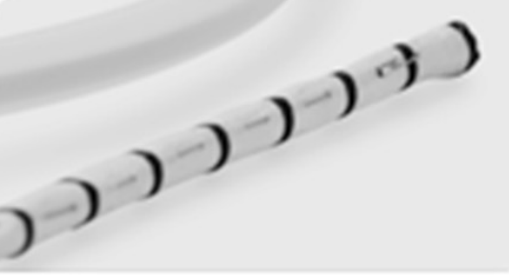

Cardioband Implant

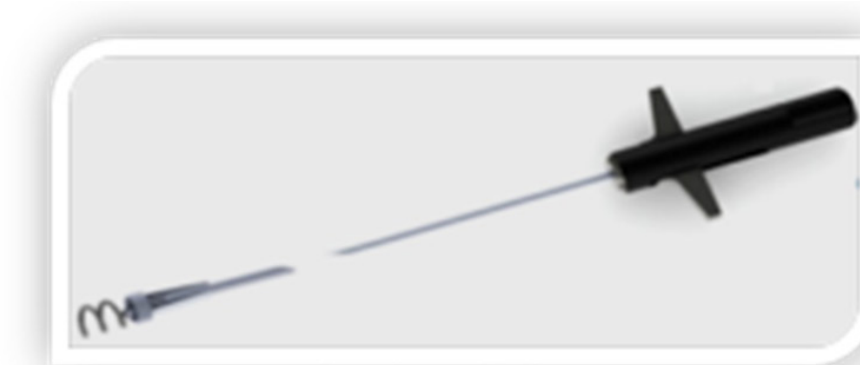

Anchor Drives

Figure 14. Components of the Cardioband System

to the $3 \mathrm{D}$ surgical view, targeting the prolapsing segment. This procedural step relies on specific technical peculiarities to confirm leaflet grasping and capture. After that, the device is finally pulled out from the LV apex and tension is adjusted under real time TEE monitoring till effective MR reduction, avoiding asymmetry of leaflets apposition. Additional chordae could be implanted by repeating the procedure. At the end of the procedure, the apical purse-strings are tied and access site closed.

\section{ANNULAR APPROACH}

Transcatheter MV annuloplasty devices, mimicking surgical annuloplasty, restore the normal ratio between the leaflet surface area and the annular area, thus improving leaflets coaptation and can be performed in selected patients as a stand-alone procedure or in one step or double steps combination with other approaches, such as Mitraclip ${ }^{\mathrm{TM}} /$ chordal implantation $^{[21,22]}$.

It should be underlined that an appealing feature of this approach is the preservation of the native valve anatomy, thus keeping the option for future MV interventions/re-repair ${ }^{[23]}$.

\section{Direct annuloplasty}

The Cardioband ${ }^{\mathrm{TM}}$ device (Edwards Lifesciences, Irvine, California, USA) is an incomplete adjustable surgical-like Dacron band which is trans-septally delivered, and implanted from anterolateral to posteromedial commissure on the posterior annulus under echocardiographic and fluoroscopic guidance.

The system is constituted by [Figure 14]: the implant and the anchors.

One of the most important aspect for procedural success is pre-interventional screening based on echocardiography and mostly on CT scan, assessing (1) technical feasibility, mainly based on the relationship between circumflex artery (CA) and posterior annulus to avoid the injury to the artery; (2) annulus sizing and thickness; and (3) the anatomy of LA and IAS.

Pre-procedural CT based planning provides: (1) the coordinates for TSP site; (2) the angle of anchor deployment; (3) the distance from the leaflets hinge point; (4) the distance from CA; and (5) expected fluoroscopic projections.

\section{Intraprocedural monitoring}

The implantation of the Cardioband ${ }^{\mathrm{TM}}$ (Edwards Lifesciences, Irvine, California, USA) needs to be monitored step by step using a combination of different imaging modalities: $2 \mathrm{D}$ and $3 \mathrm{D}$ TEE, fluoroscopy and angiography [Figure 15 and Table 2]. As pre-procedural planning is heavily dependent on CT scan, intraprocedural monitoring could be tremendously eased by the upcoming fusion imaging between real time echocardiography and pre-registered CT scan. 


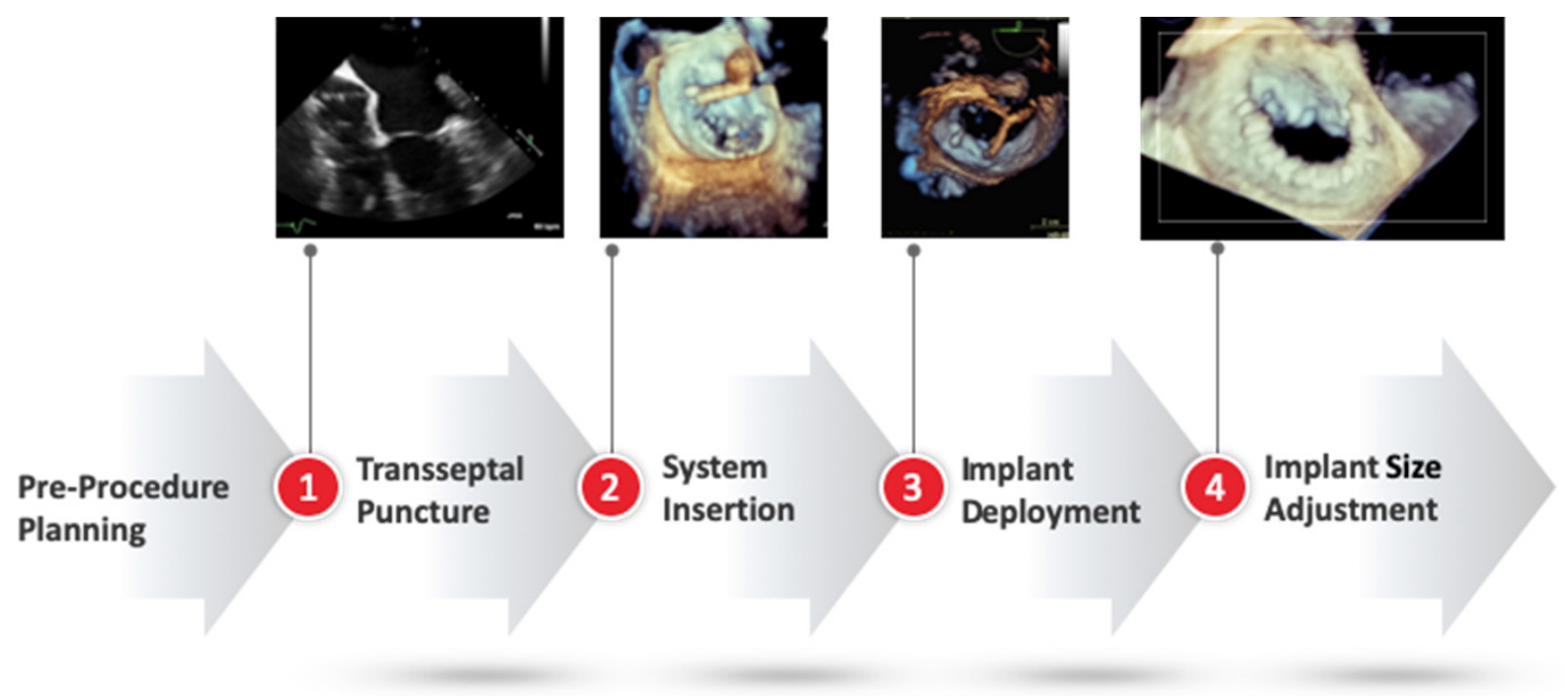

Figure 15. Procedural steps

Table 2. Imaging modality for each procedural step

\begin{tabular}{|c|c|c|c|c|}
\hline \multirow{2}{*}{\multicolumn{2}{|c|}{ Procedural Step }} & \multicolumn{2}{|l|}{ Imaging modality } & \multirow{2}{*}{ TIPS and TRICKS } \\
\hline & & \multirow{2}{*}{$\begin{array}{l}\text { Echocardiography } \\
\text { Biplane views: bicaval and SAX views } \\
\text { 3D lateral perspective of IAS } \\
\text { ME 4-chamber view with retroflexion } \\
\text { (height) }\end{array}$} & \multirow{2}{*}{$\begin{array}{l}\text { Fluoroscopy } \\
\text { AP projection } \\
\text { LAO projection }\end{array}$} & \\
\hline 1. & $\begin{array}{l}\text { Tailored patient-specific } \\
\text { trans-septal puncture }\end{array}$ & & & $\begin{array}{l}=>\text { TSP must be on top the } \\
\text { posteromedial commissure } \\
=>\text { superior and posterior location in } \\
\text { the fossa with a height of } 3.5 \mathrm{~cm} \text { to the } \\
\text { annulus (see text for details) } \\
=>\text { avoid PFO }\end{array}$ \\
\hline 2. & $\begin{array}{l}\text { Navigation of the Trans- } \\
\text { septal Sheath and Guide } \\
\text { Catheter inside the LA }\end{array}$ & $3 D$ overhead of $L A$ & LAO projection & \\
\hline 3. & $\begin{array}{l}\text { Implant Catheter } \\
\text { Placement and } \\
\text { Deployment of Anchors }\end{array}$ & $\begin{array}{l}\text { Biplane views } \\
\text { and real time Multiplanar Reconstruction } \\
3 D \text { overhead of LA }\end{array}$ & $\begin{array}{l}\text { RAO projection } \\
\text { Coronary } \\
\text { angiography }\end{array}$ & $\begin{array}{l}=>\text { the tip of the catheter should be in } \\
\text { contact with tissue along the annulus } \\
=>\text { distance from the hinge point and } \\
\text { implant angle are paramount } \\
=>\text { rule out circumflex damage }\end{array}$ \\
\hline 4. & $\begin{array}{l}\text { Implant Catheter removal } \\
\text { and SAT insertion }\end{array}$ & 3D en face view & RAO projection & $\begin{array}{l}=>\text { real time } 2 D \text { color-Doppler: balance } \\
\text { between MR reduction and iatrogenic } \\
\text { stenosis } \\
=>\text { careful evaluation of complications } \\
\text { (e.g., significant IAS shunt, pericardial } \\
\text { effusion, circumflex artery damage) }\end{array}$ \\
\hline 5. & $\begin{array}{l}\text { Implant size adjustment/ } \\
\text { cinching }\end{array}$ & $\begin{array}{l}\text { 2D color-Doppler } \\
\text { 3D color-Doppler } \\
\text { MPR Color-Doppler } \\
\text { Pressure gradient } \\
\text { MPR valve area and annular remodeling }\end{array}$ & $\begin{array}{l}\text { RAO and LAO } \\
\text { projections }\end{array}$ & \\
\hline
\end{tabular}

SAX: short axis; AP: antero-posterior; LAO: left anterior oblique; IAS: interatrial septum; PFO: patent foramen ovalis; RAO: right anterior oblique; LA: left atrium; MPR: multiplanar reconstruction

Transeptal puncture and Transseptal Sheath insertion

The optimal TSP site is pre-defined by CT planning which provides data regarding the distances from muscular part in bicaval view, from aorta in SAX-B view and the height from annular plane in fourchamber view. In particular the puncture site must be above the posteromedial commissure: this is best appreciated on the $3 \mathrm{D}$ overhead perspective of LA or en face view of the IAS from LA [Figure 16]. The height of the TSP must be $>3.5 \mathrm{~cm}$ from annular plane, as measured in four-chamber view. 

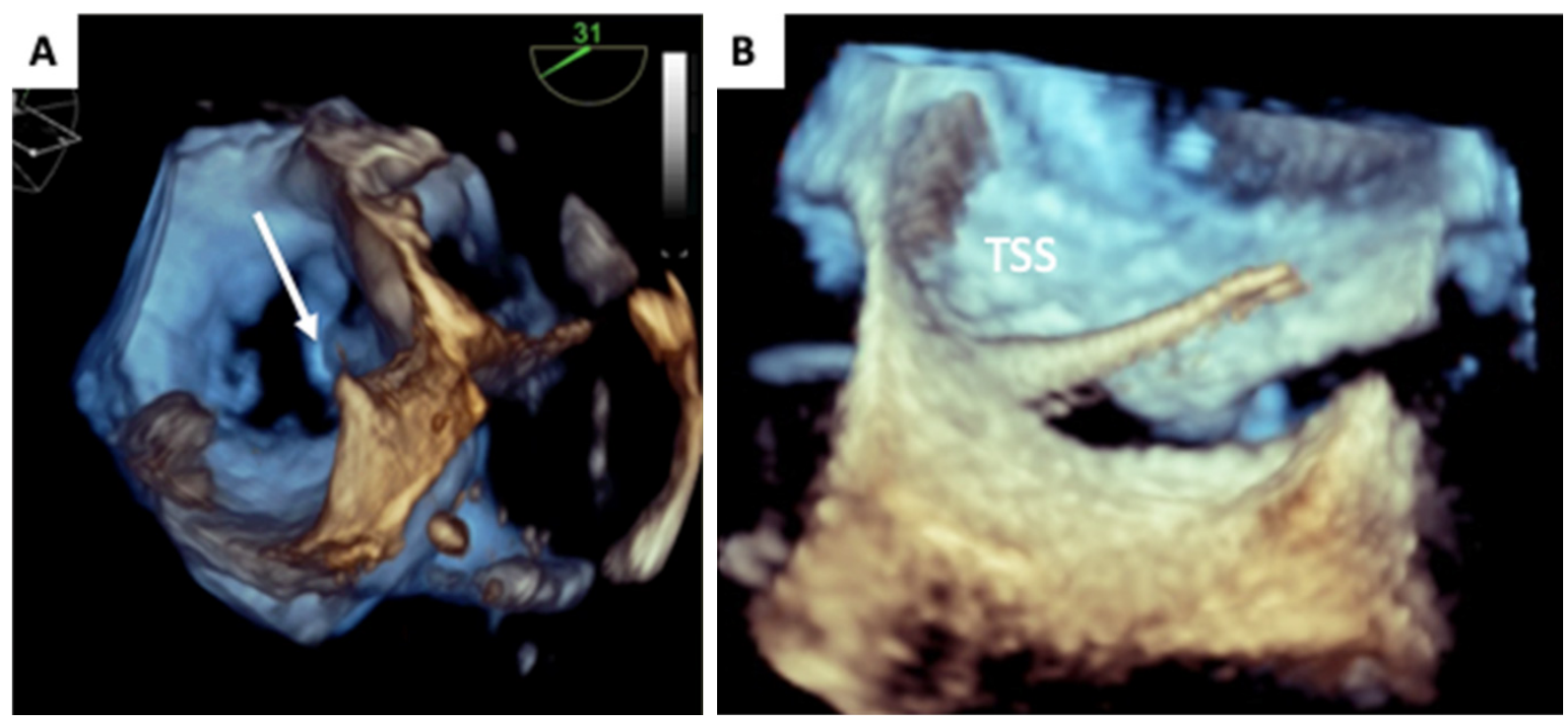

Figure 16. Transseptal puncture. A: posteromedial commissural perspective: tenting (white arrow) must be located exactly above the posteromedial commissure; B: 3D lateral perspective of LA showing the TSS across the septum (with the dilator) inside the LA. TSS: transseptal steerable sheath; LA: left atrium

\section{Navigation inside left atrium and Implant deployment}

Different 3D perspectives and fluoroscopic LAO CAU view enable visualization of the system in the different annular segments and the steering of implant delivery system (IDS) inside the LA along the posterior annulus [Figures 17 and 18]. After reaching the target point, the implant catheter (IC) is advanced to contact the annulus: several $2 \mathrm{D}$ and biplane views or $3 \mathrm{D}$ multiplanar reconstruction allow verification of the location of the tip of IC in the different annular segments, in terms of proper distance from the hinge point of posterior leaflet and device angulation in relation to the annulus.

Anchoring should be close to the leaflet hinge point in order to effectively remodel the annulus. The first anchor should be as anterior as possible, closest to the anterior trigone. After confirmation of the location with 3D overhead LA perspective, coronary angiography is performed to rule out the risk of CA injury. The anchor is then released following verification of proper anchoring through a push-and-pull test under $2 \mathrm{D}$ echocardiographic and fluoroscopic (RAO view) guidance. As the first 3 anchors operate as a root foundation for the procedure, they are implanted close to each other. The Cardioband ${ }^{\mathrm{TM}}$ implant is deployed until the radiopaque marker of the IC reaches the next marker on the implant itself. The IC tip is then navigated to the next anchoring point along the posterior annulus using echocardiographic guidance (3D overhead perspective), until the IC reaches the last anchoring site on the posterior trigone. $3 \mathrm{D}$ overhead perspective of MV is useful for guiding positioning and gross movement of the IDS along the mitral annulus, as the band is gradually deployed, anchor by anchor. Live 3D multiplanar reconstruction is of utmost importance for fine adjustment of IDS trajectory/angle in relation to the annular shelf and for fine positioning of the annulus for safe and effective anchor deployment.

\section{IDS removal and size adjustment tool insertion}

After the last anchor deployment and disconnection from the IDS, the size adjustment tool (SAT) is inserted through the transseptal steerable sheath until its distal end reaches the adjustment spool of the implant under $3 \mathrm{D}$ echocardiographic and fluoroscopic guidance.

\section{Implant size adjustment and cinching}

After SAT connection, the implant is contracted by rotation of the adjustment roller: reduction of MR severity assessed by color-Doppler and reduction of annulus size are monitored [Figure 19]. Appropriate 

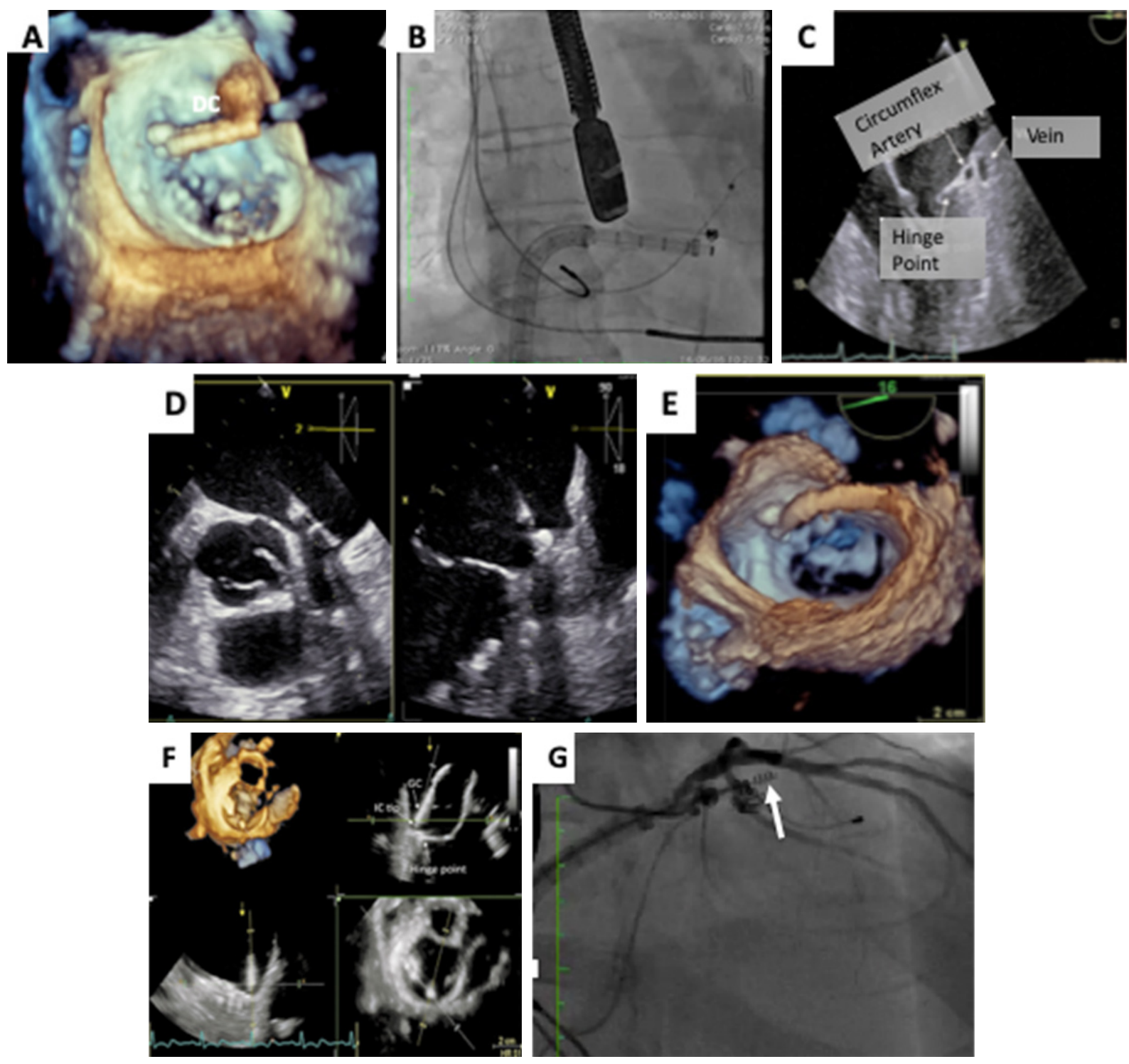

Figure 17. Navigation inside left atrium and Implant deployment. DC positioned at level of anterior commissure: A: 3D overhead perspective; B: AP fluoroscopic projection; C: echocardiographic localization of circumflex artery; D: biplane imaging for fine tip positioning on the annulus and assessing the local angle of approach to the annulus; E: 3D MV en face view showing spatial relationships during deployment of first anchors in antero-lateral commissural area; F: multiplanar reconstruction showing anchor delivering; G: coronary angiography: spatial relationship between anchor (white arrow) and circumflex artery. DC: delivery catheter; AP: antero-posterior; MV: mitral valve

implant size is a compromise between adequate MR reduction without iatrogenic mitral stenosis. The SAT is then detached leaving the implant with the desired degree of contraction.

Assessment of results and detection of complications

Following the Mitraclip ${ }^{\mathrm{TM}}$ procedure, a multi-modal approach is most appropriate to characterize and quantify residual MR. Particular to this procedure is the final assessment of mitral annular remodeling of which 3D multiplanar reconstruction seems to be the best method.

Percutaneous MV direct annuloplasty is generally a safe and effective procedure even in high-risk patients and is associated with few major complications ${ }^{[23]}$. 

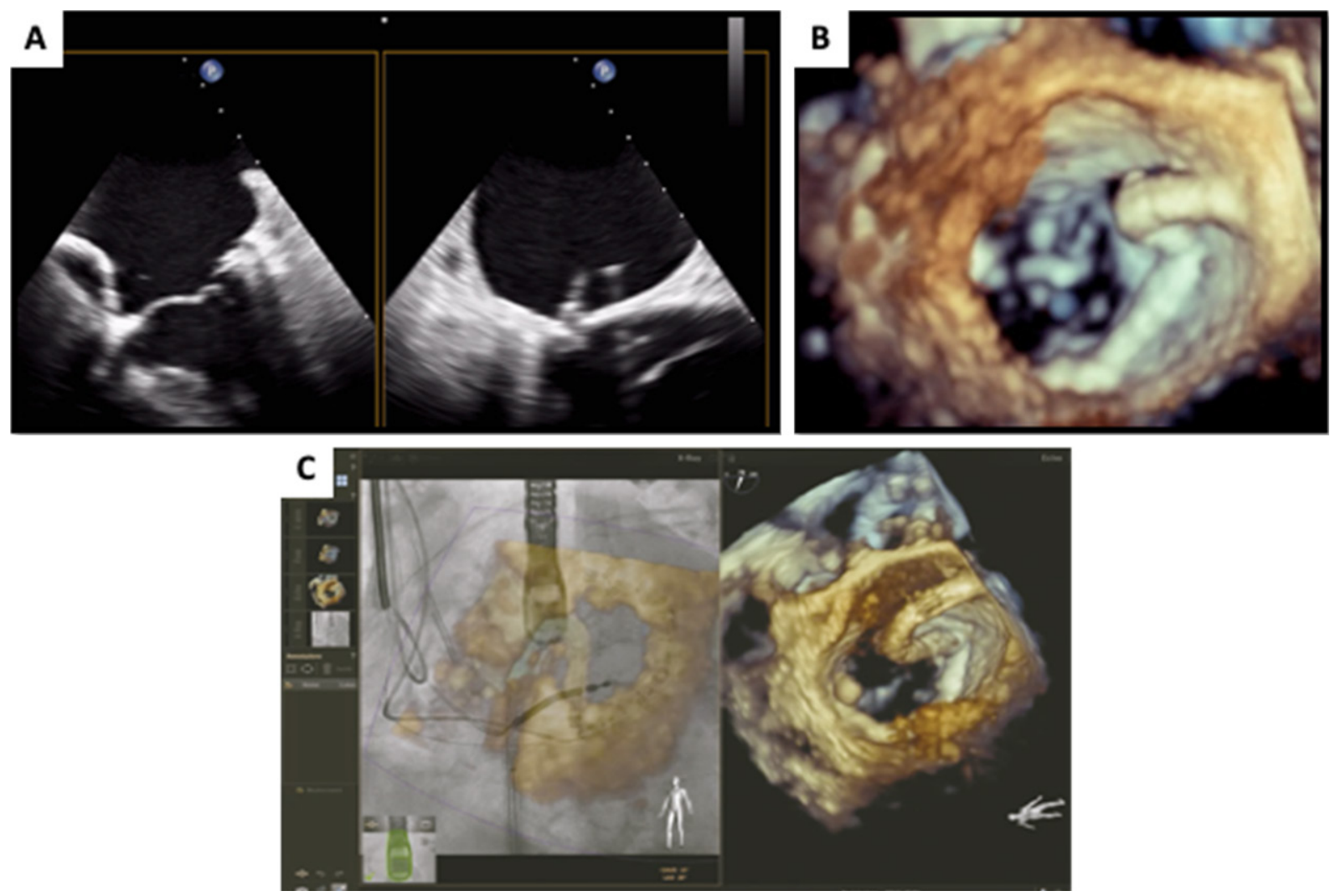

Figure 18. Last anchors deployment. A: $2 \mathrm{D}$ biplane views (starting at $60^{\circ}$ ): $2 \mathrm{D}$ echo image quality is usually sub optimal at the posteromedial commissure; B: 3D surgical view and (C) fusion imaging superimposed to the LAO CAU fluoroscopic view showing deployment of the last anchors. LAO: left anterior oblique; CAU: caudal

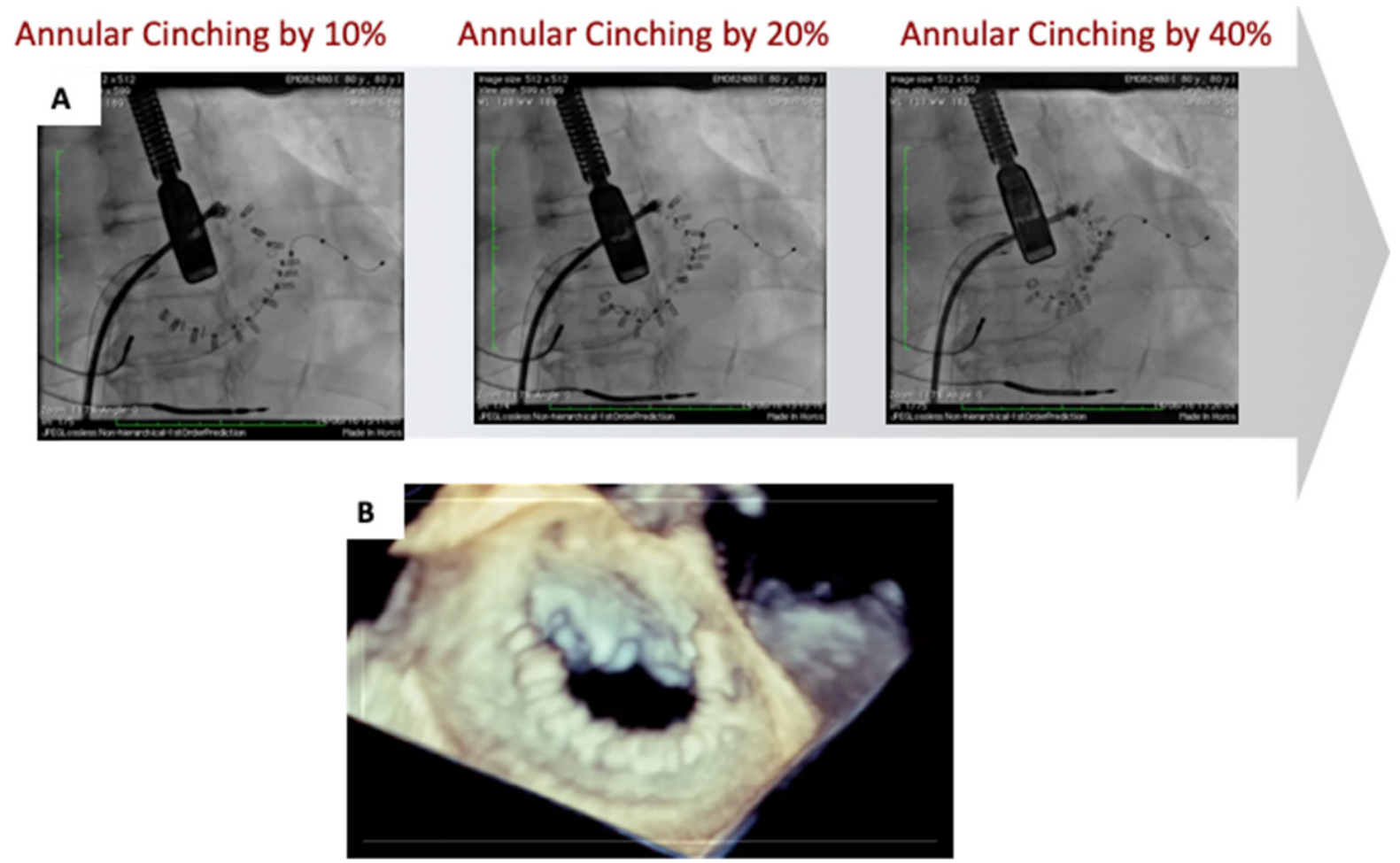

Figure 19. Cinching and final result. A: $L A O$ fluoroscopic projection at different degrees of cinching; B: $3 D$ en face view showing final result. LAO: left anterior oblique 

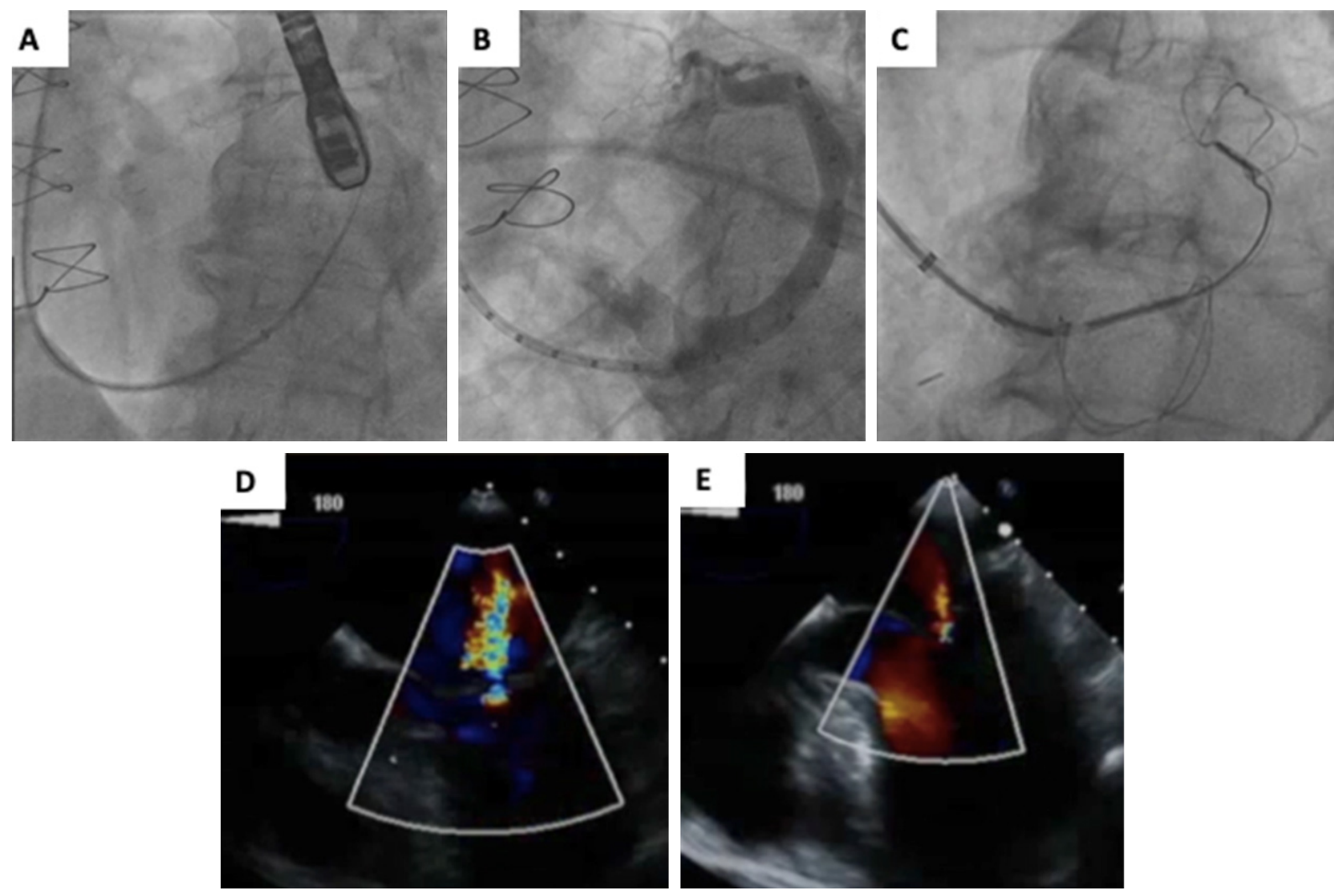

Figure 20. Procedural guidance of Coronary Sinus Annuloplasty. After cannulation of the CS, a delivery catheter is positioned distal in the $\mathrm{CS} / \mathrm{GCV}(\mathrm{A})$, as close as the antero-lateral commissure of the $\mathrm{MV}$; quantitative venography (B) and pre-procedural CT allows for selection of the appropriate implant size. After deployment of the distal anchor of the device and application of manual traction, the proximal anchor is deployed (C); reduction of the septo-lateral dimensions and MR downgrading are finally assessed by fluoroscopy and color Doppler imaging: pre-procedural (D) and post-procedural MR (E). CS: coronary sinus; GCV: great coronary vein; CT: computed tomography; MV: mitral valve; MR: mitral regurgitation

\section{Transfemoral indirect annuloplasty: coronary sinus annuloplasty}

Indirect annuloplasty through the coronary sinus re-shapes the anteroposterior MV annular dimensions to improve mitral leaflet apposition and thus coaptation, due to close relationship between the coronary sinus (CS)/great cardiac vein (GCV) and the posterior part of the MV annulus.

The Carillon ${ }^{\mathrm{TM}}$ Mitral Contour System (Cardiac Dimensions, Kirkland, WA, USA), is the most clinically tested indirect annuloplasty device, consisting of two helical anchors connected by a nitinol bridge.

The AMADEUS study reported implantation feasibility without significant MR improvement in a small population while undertaking a moderate risk of coronary complications ${ }^{[24]}$ while the TITAN trial reported successful implantation leading to MR reduction in 36 of 53 enrolled patients with subsequent LV reverse remodelling ${ }^{[25]}$.

Using the right internal jugular access, the distal anchor is positioned in the CS/GCV, and subsequent traction is applied to re-shape the septo-lateral MV annular dimensions, using a combination of fluoroscopy, as the main imaging modality, and TEE or transthoracic monitoring as adjuncts, mainly used for final procedural assessment [Figure 20]. A LAO caudal projection, the fluoroscopical short axis of the $\mathrm{MV}$, shows the CS surrounding the posterior part of the MV. 
After cannulation of the CS, a $9 \mathrm{~F}$ delivery catheter is positioned distal in the GCV, as close as the anterolateral commissure of the MV. Then the arteriovenous anatomy is characterized through occlusive venography and coronary angiography, allowing for the selection of the appropriate implant size. After the distal anchor of the device is deployed, manual traction is applied to reshape the periannular tissue. Ultimate device size and position are determined by maximal geometric reduction of the septo-lateral dimensions and reduction of MR, as assessed by fluoroscopy and TEE (mainly mid-esophageal views and 3D MV en face view). Before final release, coronary angiography rules out coronary injuries/preservation of coronary flow. The implant can be recaptured and repositioned. Procedural success strongly relies on the variable distance/relationship between the CS and the posterior MV annulus, influencing effective annular cinching.

\section{CONCLUSION}

Percutaneous MV therapies are increasingly emerging as safe alternatives for high-risk patients not suitable for conventional open-heart surgery. Intraprocedural monitoring relies on the sapient integration of fluoroscopy and echocardiography, highlighting the importance of the communication inside the cath lab between the interventional imager and the interventional cardiologist in order to perform more effective and safer procedures.

\section{DECLARATIONS}

\section{Authors' contributions}

Made substantial contributions to conception and design of the study and performed data analysis and interpretation: Ancona F, Stella S, Capogrosso C, Agricola E

Performed data acquisition, as well as provided administrative, technical, and material support: Melillo F, Ingallina $\mathrm{G}$, Boccellino A, Napolano A

\section{Availability of data and materials}

Not applicable.

\section{Financial support and sponsorship}

None.

\section{Conflicts of interest}

All authors declared that there are no conflicts of interest.

\section{Ethical approval and consent to participate}

Not applicable.

\section{Consent for publication}

A written informed consent for publication was obtained.

\section{Copyright}

(c) The Author(s) 2020.

\section{REFERENCES}

1. Konstadt SN, Louie EK, Shore-Lesserson L, Black S, Scanlon P. The effects of loading changes on intraoperative Doppler assessment of mitral regurgitation. J Cardiothorac Vasc Anesth 1994;8:19-23.

2. Lancellotti P, Tribouilloy C, Hagendorff A, et al; Scientific document committee of the european association of cardiovascular imaging. Recommendations for the echocardiographic assessment of native valvular regurgitation: an executive summary from the European Association of Cardiovascular Imaging. Eur Heart J Cardiovasc Imaging 2013;14:611-44. 
3. Alfieri O, Maisano F, De Bonis M, et al. The double-orifice technique in mitral valve repair: a simple solution for complex problems. $J$ Thorac Cardiovasc Surg 2001;122:674-81.

4. Khalique OK, Hahn RT. Role of echocardiography in transcatheter valvular heart disease interventions. Curr Cardiol Rep 2017;19:128.

5. Labrousse L, Dijos M, Leroux L, et al. Guidance of the MitraClip ${ }^{\circledR}$ procedure by 2D and 3D imaging. Arch Cardiovasc Dis 2018;111:432-40.

6. Wunderlich NC, Beigel R, Ho SY, et al. Imaging for mitral interventions: methods and efficacy. JACC Cardiovasc Imaging 2018;11:872901.

7. Agricola E. Interventional echocardiography. Edizioni Minerva Medica; 2020.

8. Sürder D, Pedrazzini G, Gaemperli O, et al. Predictors for efficacy of percutaneous mitral valve repair using the MitraClip system: the results of the MitraSwiss registry. Heart 2013;99:1034-40.

9. Herrmann HC, Kar S, Siegel R, et al; EVEREST Investigators. Effect of percutaneous mitral repair with the MitraClip device on mitral valve area and gradient. EuroIntervention 2009;4:437-42.

10. Boerlage-van Dijk K, van Riel AC, de Bruin-Bon RH, et al. Mitral inflow patterns after MitraClip implantation at rest and during exercise. J Am Soc Echocardiogr 2014;27:24-31.e1.

11. Zoghbi WA, Asch FM, Bruce C, et al. Guidelines for the evaluation of valvular regurgitation after percutaneous valve repair or replacement: a report from the American Society of Echocardiography Developed in Collaboration with the Society for Cardiovascular Angiography and Interventions, Japanese Society of Echocardiography, and Society for Cardiovascular Magnetic Resonance. J Am Soc Echocardiogr 2019;32:431-75.

12. Dietl A, Prieschenk C, Eckert F, et al. 3D vena contracta area after MitraClip $\odot$ procedure: precise quantification of residual mitral regurgitation and identification of prognostic information. Cardiovasc Ultrasound 2018;16:1.

13. Avenatti E, Mackensen GB, El-Tallawi KC, et al. Diagnostic value of 3-dimensional vena contracta area for the quantification of residual mitral regurgitation after MitraClip procedure. JACC Cardiovasc Interv 2019;12:582-91.

14. Smith T, McGinty P, Bommer W, et al. Prevalence and echocardiographic features of iatrogenic atrial septal defect after catheter-based mitral valve repair with the MitraClip system. Catheter Cardiovasc Interv 2012;80:678-85.

15. Maisano F, Franzen O, Baldus S, et al. Percutaneous mitral valve interventions in the real world: early and 1-year results from the ACCESS-EU, a prospective, multicenter, nonrandomized post-approval study of the MitraClip therapy in Europe. $J$ Am Coll Cardiol 2013;62:1052-61.

16. Feldman T, Kar S, Rinaldi M, et al; EVEREST Investigators. Percutaneous mitral repair with the MitraClip system: safety and midterm durability in the initial EVEREST (Endovascular Valve Edge-to-Edge REpair Study) cohort. J Am Coll Cardiol 2009;54:686-94.

17. Colli A, Zucchetta F, Torregrossa G, et al. Transapical off-pump mitral valve repair with Neochord Implantation (TOP-MINI): step-bystep guide. Ann Cardiothorac Surg 2015;4:295-7.

18. Colli A, Manzan E, Zucchetta F, et al. Feasibility of anterior mitral leaflet flail repair with transapical beating-heart neochord implantation. JACC Cardiovasc Interv 2014;7:1320-1.

19. Gerosa G, D’Onofrio A, Manzan E, et al. One-stage off-pump transapical mitral valve repair and aortic valve replacement. Circulation 2015;131:e430-4.

20. Gammie JS, Bartus K, Gackowski A, et al. Beating-heart mitral valve repair using a novel ePTFE cordal implantation device: a prospective trial. J Am Coll Cardiol 2018;71:25-36.

21. Mangieri A, Colombo A, Demir OM, et al. Percutaneous direct annuloplasty with edge-to-edge technique for mitral regurgitation: replicating a complete surgical mitral repair in a one-step procedure. Can J Cardiol 2018;34:1088.e1-2.

22. Latib A, Ancona MB, Ferri L, et al. Percutaneous direct annuloplasty with cardioband to treat recurrent mitral regurgitation after MitraClip implantation. JACC Cardiovasc Interv 2016;9:e191-2.

23. Maisano F, Taramasso M, Nickenig G, et al. Cardioband, a transcatheter surgical-like direct mitral valve annuloplasty system: early results of the feasibility trial. Eur Heart $J$ 2016;37:817-25.

24. Schofer J, Siminiak T, Haude M, et al. Percutaneous mitral annuloplasty for functional mitral regurgitation: results of the CARILLON Mitral Annuloplasty Device European Union Study. Circulation 2009;120:326-33.

25. Siminiak T, Wu JC, Haude M, et al. Treatment of functional mitral regurgitation by percutaneous annuloplasty: results of the TITAN Trial. Eur J Heart Fail 2012;14:931-8. 